Draft version OCtober 26, 2018

Preprint typeset using $\mathrm{LAT}_{\mathrm{E} X} \mathrm{X}$ style emulateapj v. 08/22/09

\title{
EVIDENCE OF STAR FORMATION IN LOCAL S0 GALAXIES: SPITZER OBSERVATIONS OF THE SAURON SAMPLE
}

\author{
Pasquale Temi $^{1,2}$, Fabrizio Brighenti ${ }^{3,4}$, William G. Mathews ${ }^{3}$ \\ Draft version October 26, 2018
}

\begin{abstract}
We discuss infrared Spitzer observations of early type galaxies in the SAURON sample at 24,60 and $170 \mu \mathrm{m}$. When compared with 2MASS $K s$ band luminosities, lenticular (S0) galaxies exhibit a much wider range of mid to far-infrared luminosities then elliptical (E) galaxies. Mid and far-infrared emission from E galaxies is a combination of circumstellar or interstellar emission from local masslosing red giant stars, dust buoyantly transported from the galactic cores into distant hot interstellar gas and dust accreted from the environment. The source of mid and far-IR emission in S0 galaxies is quite different and is consistent with low levels of star formation, $0.02-0.2 M_{\odot} \mathrm{yr}^{-1}$, in cold, dusty gaseous disks. The infrared $24 \mu \mathrm{m}-70 \mu \mathrm{m}$ color is systematically lower for (mostly S0) galaxies with known molecular disks. Our observations support the conjecture that cold dusty gas in some S0 galaxies is created by stellar mass loss at approximately the same rate that it is consumed by star formation, so the mass depletion of these disks by star formation will be slow. Unlike E galaxies, the infrared luminosities of S0 galaxies correlate with both the mass of molecular gas and the stellar $\mathrm{H} \beta$ spectral index, and all are related to the recent star formation rate. However, star formation rates estimated from the $\mathrm{H} \beta$ emission line luminosities $L_{H \beta}$ in SAURON S0 galaxies are generally much smaller. Since $L_{H \beta}$ does not correlate with $24 \mu \mathrm{m}$ emission from dust heated by young stars, optical emission lines appear to be a poor indicator of star formation rates in SAURON S0 galaxies. The absence of $\mathrm{H} \beta$ emission may be due to a relative absence of $\mathrm{OB}$ stars in the initial mass function or to dust absorption of $\mathrm{H} \beta$ emission lines.
\end{abstract}

Subject headings: galaxies: elliptical and lenticular; galaxies: ISM; infrared: galaxies; infrared: ISM

\section{INTRODUCTION}

In the discussion below we extend our earlier analysis of Spitzer infrared observations of elliptical galaxies (E) (Temi, Brighenti Mathews, 2007a) to include lenticular galaxies (S0). The data set we consider here is further restricted to 31 early type E (19) and S0 (12) galaxies that have been observed by both Spitzer and SAURON. The SAURON sample is generally representative and has received much attention from observers at all wavelengths, allowing detailed comparisons of Spitzer infrared observations with many different galactic attributes: stellar populations, nature and extent of cold gas, dust content, etc.

Our principal objective is to explore evidence of recent star formation in local S0 galaxies at mid and far infrared wavelengths and to contrast this with the apparent lack of measurable current star formation in the sample E galaxies. Many S0 galaxies in the SpitzerSAURON sample have attributes consistent with recent star formation: significantly larger mid and far infrared luminosities, large masses of molecular gas, and younger stellar ages as inferred from optical absorption line spectra. Spitzer-SAURON S0 galaxies having the largest farinfrared luminosities are known to contain rotationally

${ }^{1}$ Astrophysics Branch, NASA/Ames Research Center, MS 2456, Moffett Field, CA 94035

2 Department of Physics and Astronomy, University of Western Ontario, London, ON N6A 3K7, Canada. ptemi@mail.arc.nasa.gov

${ }^{3}$ University of California Observatories/Lick Observatory, Board of Studies in Astronomy and Astrophysics, University of California, Santa Cruz, CA 95064 mathews@ucolick.org

${ }^{4}$ Dipartimento di Astronomia, Università di Bologna, via Ranzani 1, Bologna 40127, Italy fabrizio.brighenti@unibo.it supported disks of molecular hydrogen with masses $\gtrsim 10^{8}$ $M_{\odot}$. Using the $24 \mu$ emission as a guide, stars are forming in many sample lenticular galaxies at very modest rates, $0.02-0.2 M_{\odot} \mathrm{yr}^{-1}$. For some of these S0 galaxies this level of star formation is comparable to the rate that gas is globally supplied from an old population of evolving stars, suggesting a quasi-steady state. In their optical spectra our sample S0 galaxiess exhibit Balmer absorption lines from young A-F stars, but the Balmer line emission from ionized gas associated with more massive O-B stars can be lower than expected. In addition, when star formation rates are estimated using standard empirical procedures, the rates determined from $\mathrm{H} \beta$ emission line luminosities in Spitzer-SAURON S0 galaxies can be much less than those derived from $24 \mu \mathrm{m}$ luminosity. The cold, dusty molecular disks in some S0 galaxies may originate from stellar mass loss, may be a vestige of a more substantial disk during a previous incarnation as a more normal spiral galaxy, or may have been acquired by a merger event.

\section{THE SAURON-SPITZER SAMPLE}

In Table 1 we list 34 (non-dwarf) $\mathrm{E}$ and S0 galaxies in the SAURON sample that are also in the public Spitzer archive. This table contains additional details about each galaxy that will be discussed below. The SAURON sample is not complete but was chosen to include nearby early type galaxies representing a limited range of absolute magnitude, ellipticity, environments, etc. as described in detail by de Zeeuw et al. (2002). The Spitzer-SAURON sample that we discuss here includes additional imponderable attributes imposed by infrared observers who contributed to the Spitzer archive. 


\section{OBSERVATIONS AND REDUCTION}

Far infrared data presented here were obtained with the Multiband Imager Photometer (MIPS) (Rieke et al. 2004) on board the Spitzer Space Telescope (Werner et al. 2004) in three wavebands centered at 24, 70 and $160 \mu \mathrm{m}$. These data, collected from the Spitzer public archive, do not form an homogeneous and uniform data set in terms of image depth and observing mode. Table 1 lists the program ID and PI name of the original Spitzer observing program from which sample galaxies have been selected. The reader is referred to these programs to obtain details on the observing modes and imaging strategy, as well as the on-source integration time for each target in the sample. As an example, Virgo Cluster galaxies recorded under the guaranteed time program (PID 69, PI G. Fazio) reach a relatively low sensitivity of $0.5 \mathrm{MJy} / \mathrm{sr}$ and $1.1 \mathrm{MJy} / \mathrm{sr}(1 \sigma)$ at 70 and $160 \mu \mathrm{m}$, respectively, while other galaxies (i.e. PID 20171, PI P. Temi) have deeper maps at a sensitivity level of only $0.12 \mathrm{MJy} / \mathrm{sr}$ and $0.3 \mathrm{MJy} / \mathrm{sr}$ for the same two wavebands. The SINGS data are recorded in MIPS scan mode, covering a very large sky area $\left(30^{\prime} \times 10^{\prime}\right)$, incorporating two separated passes at each source location. SINGS images correspond to maps with intermediate sensitivity. Apart from the SINGS observations, data have been acquired in MIPS photometry mode, allowing appropriate coverage of the sources and their extended emission.

Reduction of Spitzer-SAURON data follows the same procedure used in Temi, Brighenti \& Mathews (2007a). Here we briefly summarize the basic processes involved in the reduction. We started with the Basic Calibrated Data (BCD) products from the Spitzer Science pipeline (version 16.1) to construct mosaic images for all objects. Final calibrated images have been produced using the MOPEX (Mosaicking and Point-source Extraction) package developed at the Spitzer Science Center (Makovoz et al. 2006). MOPEX includes all the functions and steps necessary to process BCD data into corrected images and co-add them into a mosaic. The major MOPEX pipeline used was the mosaic pipeline which consists of a number of individual modules to be run in sequence to properly perform the reduction. We refer the reader to the MOPEX webpage for a detailed description of each module. Since the data sets presented here have been acquired in different observing modes, the modules chosen in build-up the reduction flow and their parameter setup have been carefully selected to properly remove mode dependent artifacts in the final mosaic (outlier detection and median filtering).

If foreground stars and background galaxies were present in the nal mosaiced images they were edited before flux extraction was performed. These were identified by eye and cross-checked using surveys at other wavelengths (Digital Sky Survey and 2MASS). Flux densities were extracted from apertures that cover the entire optical disk (R25). Sky subtraction was performed by averaging values from multiple apertures placed around the target, avoiding any overlap with the faint extended emission from the galaxy. Statistical uncertainties related to sky subtraction are usually less than $1 \%$ but can be appreciable (tens of percent) for faint sources. Observed infrared flux densities for each galaxy are listed in Table 2. Columns $2-4$ contain the total flux $F_{\lambda} \Delta \lambda$ (mJy) at 24, 70 and $160 \mu \mathrm{m}$ within each MIPS passband with respective widths $\Delta \lambda=5.3,19$ and $34 \mu \mathrm{m}$.

Systematics in the MIPS calibration result in fluxes uncertain at the $10 \%$ level at $24 \mu \mathrm{m}$ and $20 \%$ at 70 and 160 $\mu \mathrm{m}$. The uncertainties listed in Table 2 do not include the systematic uncertainties. Aperture corrections for extended sources were applied to the fluxes as described in the Spitzer Observer's manual. The corresponding MIPS luminosities are $L_{\lambda}=F_{\lambda} \Delta \lambda \cdot 4 \pi D^{2}$ where distances $D$ are from Table 1 .

\section{OBSERVATIONS}

\subsection{Star Formation Rates from 24 Micron Emission}

Figure 1 shows the relationship between the 2MASS $K s$ band luminosities of galaxies in Table 1 and their MIPS passband luminosities at 24, 70 and $160 \mu \mathrm{m}$. The $K s$-band luminosities of E galaxies correlate tightly with $L_{24}$, but scatter dominates the correlations at 70 and $160 \mu \mathrm{m}$. These results are similar to those discussed by Temi, Brighenti \& Mathews (2007a) for a larger sample of E galaxies. Since E galaxies typically have de Vaucouleurs surface brightness profiles at $24 \mu \mathrm{m}$ (Temi, Brighenti \& Mathews 2008), we conclude that most of the $24 \mu \mathrm{m}$ emission originates in circumstellar dust around old red giant stars. However, Temi, Brighenti \& Mathews (2007a,b) argue that E galaxy emission at 70 and $160 \mu \mathrm{m}$ arises from colder, truly interstellar dust. But the stochastic variation that produces the scatter in the lower panels of Figure 1 is thought to be related to stochastic AGN energy releases from the central black hole that heats dusty gas in galactic cores which is then buoyantly transported out $5-10 \mathrm{kpc}$ into the hot gas atmospheres within these galaxies (Temi, Brighenti \& Mathews 2007a,b). Only for ellipticals with the lowest $L_{70}$ and $L_{160}$ can the emission be explained by dusty mass loss from local stars. Rare E galaxies that are unusually luminous at 70 and $160 \mu \mathrm{m}$ probably have experienced recent mergers, but, as discussed below, there is no strong evidence for recent mergers in the SAURON sample E galaxies. Note that the vertical scatter of S0 infrared luminosities in Figure 1 significantly exceeds that of the E galaxies at all wavelengths. This is one of the strikingly different properties of S0 galaxies that we emphasize here.

In Figure 2 the infrared luminosities are normalized by $L_{K s}$ (in solar unit) and the correlations at $24 \mu \mathrm{m}$ for $\mathrm{E}$ galaxies becomes much flatter. A least square fit to $24 \mu \mathrm{m}$ emission from $\mathrm{E}$ galaxies in Figure 2 gives

$$
L_{24}=10^{29.4 \pm 0.5} L_{K s}^{1.01 \pm 0.04} \quad \mathrm{erg} \mathrm{s}^{-1} .
$$

Evidently the nearly constant $\log \left(L_{24} / L_{K s}\right)=29.4$ for E galaxies represents the standard infrared SED of old stellar populations and their associated circumstellar dust. We show below that the departure of individual $\mathrm{E}$ galaxies from this relation are uncorrelated with $\mathrm{H} \beta$ emission line luminosity. Therefore, it is unlikely that the small variations of either infrared or optical line emission in SAURON E galaxies are related to appreciable star formation or recent mergers with star-forming dwarf galaxies.

While a few S0 galaxies appear to have old stars with little or no current star formation, as in many E galaxies, others have significantly larger $L_{24} / L_{K s}$ which we inter- 
pret as evidence of a subpopulation of younger stars. We imagine that interstellar dust in cold (possibly rotationally supported) gas is heated by massive, hot stars that recently formed from this gas. The large vertical scatter in the S0 galaxies at all three wavelengths in Figures 1 and 2 suggests that the interstellar dust in these galaxies has a much different origin and environment than far-IR emitting dust in E galaxies.

In their study of star-forming galaxies Calzetti et al. (2007) propose a relation between the specific $24 \mu \mathrm{m} \mathrm{lu}-$ minosity, $\left(\lambda L_{\lambda}\right)_{24} \equiv \lambda_{24} L_{\lambda_{24}}$, and the star formation rate,

$\operatorname{SFR}\left(M_{\odot} \mathrm{yr}^{-1}\right)=1.24 \times 10^{-38}\left[\left(\lambda \mathrm{L}_{\lambda}\right)_{24}\left(\operatorname{erg~s}^{-1}\right)\right]^{0.8850}$.

that can be used to find the SFR from global observations of $\left(\lambda L_{\lambda}\right)_{24}$. Values of $\left(\lambda L_{\lambda}\right)_{24}$ for our sample galaxies are plotted against $L_{K s}$ in Figure 3 .

We shall assume that this $\operatorname{SFR}-\left(\lambda L_{\lambda}\right)_{24}$ relation from Calzetti et al. holds for the $\mathrm{S} 0$ galaxies in Figure 3, but a correction must be made for the relatively strong $24 \mu \mathrm{m}$ contribution from old stars in these early type lenticular galaxies. Unlike the star-forming galaxies considered by Calzetti et al, where almost all of the $24 \mu \mathrm{m}$ emission evidently comes from interstellar dust irradiated by young hot stars, much or most of the $24 \mu \mathrm{m}$ emission from E and S0 galaxies originates in circumstellar dust surrounding old, mass-losing red giant stars. To remove this background $24 \mu \mathrm{m}$ emission, we determine the SFR from the $\left(\lambda L_{\lambda}\right)_{24}$ emission in excess of that from the old stellar populations in our sample E galaxies collectively assumed to have $S F R=0$. The correlation between the specific stellar $24 \mu \mathrm{m}$ luminosity for old E galaxies and $L_{K s}$

$$
\log \left(\lambda L_{\lambda}\right)_{24, \text { corr }}=(1.01 \pm 0.05) \log L_{K s}+30.1 \pm 0.5,
$$

is shown as a dashed line in Figure 3. Therefore to estimate the star formation rate in S0 galaxies that lie significantly above this correlation in Figure 3, we enter the excess luminosity

$$
\Delta\left(\lambda L_{\lambda}\right)_{24}=\left(\lambda L_{\lambda}\right)_{24}-\left(\lambda L_{\lambda}\right)_{24, \text { corr }}
$$

in the SFR- $\left(\lambda L_{\lambda}\right)_{24}$ relation above. The resulting SFRs for galaxies with positive $\Delta \log \left(\lambda L_{\lambda}\right)_{24}$ are listed in the last column of Table 2. If $S F R=0$ is assumed for $\mathrm{E}$ galaxies, the mean scatter among the SFRs evaluate for elliptical galaxies in the upper section of Table 2, 0.014 $M_{\text {odot }} \mathrm{yr}^{-1}$, suggests that the star formation rates for S0 galaxies (in the bottom section) are uncertain to about $\pm 0.02 M_{\text {odot }} \mathrm{yr}^{-1}$. The SFRs for these local S0 galaxies are all less than $1 M_{\odot} \mathrm{yr}^{-1}$.

If the 70 and $160 \mu \mathrm{m}$ emission from S0 galaxies is due to the same process that emits at $24 \mu \mathrm{m}$, such as star formation, we would expect the 70 and $160 \mu \mathrm{m}$ luminosities to correlate with $24 \mu \mathrm{m}$ residuals $\Delta \log \left(\lambda L_{\lambda}\right)_{24}$. This correlation is shown in Figure 4. Evidently, the same young stars in S0 galaxies that heat nearby interstellar dust grains to emit at $24 \mu \mathrm{m}$ also heat more distant grains that emit at longer wavelengths. However, when the $24 \mu \mathrm{m}$ residuals for S0 galaxies are plotted against 70 and $160 \mu \mathrm{m}$ luminosities that are normalized with the $K s$-band luminosities, as in Figure 5, the correlation is greatly improved. This figure suggests that $L_{F I R} / L_{K s}$ is likely to be an equally good measure of the star formation rate in $\mathrm{S} 0$ galaxies as the $24 \mu \mathrm{m}$ residuals. Since $\log \left(L_{B} / L_{K s}\right)$ (from Table 1$)$ does not increase systematically with $24 \mu \mathrm{m}$ residuals or $\operatorname{SFR}(24 \mu \mathrm{m})$, we conclude that the low level of star formation in S0 galaxies produces little observable additional $B$-band (or $K s$-band) emission. Consequently, $L_{K s}$ is a good measure of the mass of old stars and is insensitive to the small SFR in these S0 galaxies.

But Figure 5 informs us about a deeper relationship between star formation, nuclear molecular disks, and the mass of old stars in S0 galaxies. Young, Bureau \& Cappellari (2008) have studied compact CO-emitting molecular disks in four SAURON S0 galaxies: NGC 3032, 4150, 4459 and 4526. These disks typically have kpcsized radii, total masses $0.5-5 \times 10^{8} M_{\odot}$ and mean surface densities $100-200 M_{\odot} \mathrm{pc}^{-2}$. In NGC 3032, the S0 with the largest apparent star formation rate in Figure 5 , the molecular disk (disk radius: $1.5 \mathrm{kpc}$, mass: $5 \times 10^{8}$ $\left.M_{\odot}\right)$ is counterrotating and must have been acquired by a galaxy merger in the past. The smaller molecular disk in NGC 4150 (disk radius: $0.5 \mathrm{kpc}$, mass: $0.55 \times 10^{8} \mathrm{M}_{\odot}$ ) is kinematically oriented with a dusty disk that obscures optical light. However, NGC 4150 contains a counterrotating core of younger stars having a spatial orientation that differs curiously from that of the molecular gas and rotates in the opposite sense. This suggests that the molecular disk in NGC 4150 also resulted from one or more recent mergers.

In the remaining two S0 galaxies observed by Young, Bureau \& Cappellari (2008) - NGC 4459 (disk radius: $0.67 \mathrm{kpc}$, mass: $1.6 \times 10^{8} M_{\odot}$ ) and NGC 4526 (disk radius: $1.1 \mathrm{kpc}$, mass: $\left.5.7 \times 10^{8} M_{\odot}\right)$ - the molecular disks are well-aligned with the rotating system of old stars. These appear to be more normal S0 galaxies. NGC 4459 and 4526, together with NGC 3156 and 3489 , form a tight group of four S0 galaxies in Figure 5 having nearly the same $L_{F I R} / L_{K s}$ and $24 \mu \mathrm{m}$ residuals, but with SFRs greater than normal elliptical galaxies. It is remarkable that among these four S0 galaxies both $L_{F I R}$ and $L_{K s}$ vary by about an order of magnitude yet they share the same location in Figure 5. Since the mass of old stars increases with $L_{K s}$ and $L_{F I R}$ depends on the total mass of dust and star-forming cold gas, it is possible that the star-forming gas in these S0 galaxies is being resupplied by mass ejected from old evolving stars. To explore this possibility, as $L_{K s}$ varies over the range $10^{10}-10^{11}$ (Figure 3 ), the rate that mass is ejected from old stars varies by about $\dot{M}_{*} \approx 0.01-0.1$ $M_{\odot} \mathrm{yr}^{-1}$ where we assume $\left(M_{*} / L_{K s}\right)=0.60$ (Bell et al. 2003) for an old stellar population with a Kroupalike IMF and $d M_{*} / d t \approx 0.15 M_{*} /\left(10^{11} M_{\odot}\right) M_{\odot *} \mathrm{yr}^{-1}$ (Mathews 1989). Within the uncertainties involved, we see that the mass supply from old red giant stars in these four star-forming SAURON S0 galaxies $\left(\dot{M}_{*} \approx 0.01-0.1\right.$ $\left.M_{\odot} \mathrm{yr}^{-1}\right)$ is nearly equal to the SFR estimated from the $24 \mu \mathrm{m}$ dust emission. Therefore it is possible that the internal production of gas in these four S0 galaxies maintains rotationally supported disks of cold dusty gas that are forming young stars at the same rate, keeping the total mass of cold gas and dust rather constant with time.

The two S0 galaxies with the largest $24 \mu \mathrm{m}$ residuals 
and $L_{F I R} / L_{K s}$ in Figure 5 - NGC 3032 and 4150 - have evidently acquired cold gas and dust in recent mergers, and their current star formation rates exceed the rate that mass is supplied by stellar mass loss. As star formation proceeds in these galaxies, we speculate that they may evolve downward in Figure 5 (NGC $3032 \rightarrow$ NGC $4150 \rightarrow$ NGC 3156) until they reach the slowly-evolving, metastable situation described above for the four galaxies including NGC 3156 where the SFR nearly balances the rate of stellar mass loss. This decreasing trend of star formation in cold nuclear disks may extend further to the only two elliptical galaxies in our sample known to contain molecular gas - NGC 2768 and 4278 - which also have the largest $L_{F I R} / L_{K s}$ among the E galaxies in Figure 5. When the stellar mass loss becomes comparable to the SFR, progress along this downward trend in Figure 5 is expected to become slower. Finally, since the stellar mass loss rate in an old single stellar population decreases with time $\left(\propto t^{-1.3}\right)$, we speculate that the oldest merger-free S0 galaxies are those in Figure 5 that have joined the cloud of elliptical galaxies in which the cold nuclear disks have no observable $\mathrm{CO}$ or far-IR emission.

As seen in the infrared color-color plot in Figure 6 the infrared color $\log \left(L_{24} / L_{70}\right)$ differs for those galaxies in Figure 5 that are known to contain molecular gas. Since these mildly star-forming galaxies have the lowest $\log \left(L_{24} / L_{70}\right)$ colors of the entire Spitzer-SAURON sample, this color could be used to identify other E and S0 galaxies that contain similar molecular disks.

\subsection{Correlations of $24 \mu m$ Emission with $H \beta$ Index and Molecular Mass}

From their analysis of SAURON galaxies McDermid et al. (2006) showed that the mean stellar age of S0 galaxies is systematically much less than that of $\mathrm{E}$ galaxies. We now show that this result is consistent with our observations at MIPS wavelengths. Young stellar ages correspond to larger $\mathrm{H} \beta$ Lick (absorption) indices in optical spectra. In Figure 7 we show that the optical $\mathrm{H} \beta$ index in S0 galaxies (evaluated within $R_{e} / 8$ ) correlates positively with bandpass luminosities at 24,70 and $160 \mu \mathrm{m}$, suggesting that a substantial fraction of this infrared radiation comes from interstellar dust irradiated and heated by young stars.

The correlations in Figure 7 show that the contribution of young stars to the $\mathrm{H} \beta$ index in S0 galaxies increases with $L_{F I R} / L_{K s}$ since this latter ratio correlates with the $24 \mu \mathrm{m}$ residuals (Fig. 5) and the SFR. We expect an even stronger correlation if $\mathrm{H} \beta$ is plotted against the $24 \mu \mathrm{m}$ residuals. This expectation is confirmed in the upper panels of Figure 8 for $\mathrm{H} \beta$ indices determined within either $R_{e} / 8$ or $R_{e}$ of the galactic centers. S0 galaxies with $\mathrm{H} \beta \gtrsim 2$ contain systematically younger stars. Further confirmation that $\Delta \log \left(\lambda L_{\lambda}\right)_{24}$ is related to the star formation rate is provided by the lower panel of Figure 8 , showing that the mean stellar age from Table 1 decreases with increasing residuals in Figure 3. The three E galaxies in Figure 8 with mean stellar ages less than 5 Gyrs - NGC 2768 and 5831 - are somewhat anomalous in not having larger residuals $\Delta \log \left(\lambda L_{\lambda}\right)_{24}$.

While observations of the HI gas mass in our SpitzerSAURON sample are too sparse to consider here, more observations are available of their molecular mass
(Combes et al. 2007). Figures 9 and 10 show that the molecular mass $M_{H_{2}}$ in S0 galaxies increases with all Spitzer infrared passband luminosities $L_{F I R}$ and also with $L_{F I R} / L_{K s}$, although the scatter in Figure 10 is rather large (see also Combes et al. 2007, who found a similar correlation using IRAS data). A spurious unit slope correlation in Figure 9 could result in errors in the distances to the galaxies and this may account for the degrated correlation in Figure 10. Postive correlations in these Figures would be expected if the emitting dust in S0 galaxies is embedded inside molecular gas in which young stars are forming. The two elliptical galaxies containing molecular gas in these figures, NGC 2768 and 4278, have small positive residuals in Figure 3, but are not otherwise exceptional.

\subsection{Disparity between SFR from $24 \mu m$ and $H \beta$ Emission Luminosities}

One of the many advantages of SAURON integral field spectroscopy are improved measurements of the total luminosity of optical emission lines. In Figure 11 we plot the total luminosity in $\mathrm{H} \beta$ emission against far infrared luminosity in each MIPS passband normalized with the $K s$ band luminosity. E and S0 galaxies appear to behave in a different fashion in Figure 11. In our Spitzerbased sample of $\mathrm{E}$ galaxies we found positive correlations between the observed $\mathrm{H} \beta$ emission line flux and the bandpass flux $F_{F I R}$ at all three wavelengths, 24, 70 and $160 \mu \mathrm{m}$ (Temi, Brighenti \& Mathews 2007). However, when the FIR luminosity is normalized with $L_{K s}$, as in Figure 11, the correlation between the $\mathrm{H} \beta$ emission luminosity and $L_{24} / L_{K s}$ disappears for the E galaxies. This indicates that the $K s-24 \mu \mathrm{m}$ color is part of the normal SED of an old stellar population and that the $\mathrm{H} \beta$ emission is uncorrelated with this color. At 70 and $160 \mu \mathrm{m}$, however, E galaxy $L_{H \beta}$ correlates with $L_{F I R} / L_{K s}$. These correlations are consistent with the analysis of Temi, Brighenti \& Mathews (2007a,b) in which both $\mathrm{H} \beta$ and FIR emission arise from buoyantly transported cooling regions widely dispersed in the hot interstellar gas of E galaxies.

By contrast, the $\mathrm{H} \beta$ emission from $\mathrm{S} 0$ galaxies in Figure 11 appears not to correlate with $L_{70} / L_{K s}$ or $L_{160} / L_{K s}$ and exhibits larger overall $L_{F I R} / L_{K s}$ than the E galaxies. Evidently $\mathrm{H} \beta$ emission from S0 galaxies in the SAURON sample is generally unrelated to far infrared emission from dust. The absence of this correlation for S0 galaxies may seem surprising since massive young stars are the likely source of both optical line emission and dust heating with subsequent far-IR emission, as in the Milky Way disk. Perhaps some of the $\mathrm{H} \beta$ emission is absorbed by dust.

In the top panel of Figure 12 we show this result in a different way by plotting the $24 \mu \mathrm{m}$ residuals in Figure 3 against the $\mathrm{H} \beta$ emission line luminosity. The absence of any correlation for E galaxies indicates that there is no coordinated enhancement of emission from heated dust or optical emission lines that would be expected from star formation or from a recent merger with a starforming galaxy containing gas and dust. This result is consistent with the finding of Temi, Brighenti \& Mathews (2005) that E galaxies with mean stellar ages (from Balmer indices) less than about 5 Gyrs often show no measurable enhancement at $24 \mu \mathrm{m}$. For E galaxies the 
$\mathrm{H} \beta$ emission is clearly unrelated to star formation. A low level of $\mathrm{H} \beta$ emission is always expected in $\mathrm{E}$ galaxies from stellar mass loss into the hot interstellar gas that is ionized by hot post-AGB stars until it thermally merges into the hot interstellar phase. Larger, more stochastic contributions to optical line emission in $\mathrm{E}$ galaxies can result from cooling dusty hot gas that has been buoyantly driven out of E galaxy cores by low energy AGN activity (Temi, Brighenti, \& Mathews 2007a,b). (Mergers as a source of strong E galaxy dust emission at 70 and $160 \mu \mathrm{m}$ are also possible, particularly when there is additional morphological or dynamical evidence of a merger event.)

As before, S0 galaxies in the top panel of Figure 12 show no obvious correlation between $L_{H \beta}$ and $24 \mu \mathrm{m}$ residuals from Figure 3 . The correlations of $24 \mu \mathrm{m}$ residuals $\Delta\left(\lambda L_{\lambda}\right)_{24}$, and therefore also $\operatorname{SFR}(24 \mu \mathrm{m})$, with both the stellar $\mathrm{H} \beta$ index (Figure 8 ) and the mass of molecular gas (lower panel of Figure 12) confirm that $24 \mu \mathrm{m}$ emission is likely to be a good measure of star formation in our sample S0 galaxies. But the lack of a correlation between $L_{H \beta}$ and $24 \mu \mathrm{m}$ residuals in SAURON S0 galaxies suggests that the standard observational method of determining the SFR from optical emission lines (e.g. Kennicutt 1998; Calzetti 2007) is inaccurate or inappropriate for these galaxies which have relatively low levels of star formation.

According to Calzetti (2007), the current star formation rate can be determined from $\mathrm{H} \beta$ emission line luminosities using

$$
\operatorname{SFR}(H \beta)=1.51 \times 10^{-41} L_{H \beta} \quad\left(\operatorname{ergs~s}^{-1}\right)
$$

where we assume $L_{H \alpha}=2.86 L_{H \beta}$ since accurate $H_{\alpha}$ line luminosities cannot be measured with SAURON. (While this Case $\mathrm{B} H \alpha / H \beta$ ratio is not expected to hold in the presence of dust absorption, it is unlikely to be seriously in error for escaping line radiation.) The $\operatorname{SFR}(\mathrm{H} \beta)$ in equation 5 is derived using a Kroupa-like IMF extending to $120 M_{\odot}$. Figure 13 shows that $\operatorname{SFR}(\mathrm{H} \beta)$ is much less than $\operatorname{SFR}(24 \mu \mathrm{m})$ for many of our SAURON sample galaxies. For galaxies with the largest $\operatorname{SFR}(24 \mu \mathrm{m})-$ NGC 3032, 4526 and $4459-\mathrm{SFR}(\mathrm{H} \beta)$ is an order of magnitude smaller than $\operatorname{SFR}(24 \mu \mathrm{m})$. These are also the three galaxies in Table 1 with the largest masses of molecular gas, $1.6-5 \times 10^{8} M_{\odot}$.

Calzetti et al. (2007) also suggest a means of determining the star formation rate in the presence of dust extinction that employs both Balmer line and $24 \mu \mathrm{m}$ luminosities, $\operatorname{SFR}(24 \mu \mathrm{m}, \mathrm{H} \beta)$. However, we find that $\operatorname{SFR}(24 \mu \mathrm{m}, \mathrm{H} \beta)$ is very nearly identical to $\operatorname{SFR}(24 \mu \mathrm{m})$, i.e. the optical line emission is too low to affect the SFR from $24 \mu \mathrm{m}$ alone.

If star formation occurs in intermittent bursts separated by more than the main sequence lifetimes of $\mathrm{OB}$ stars, $\sim 10^{7} \mathrm{yrs}$, the SFR from HII emission line luminosities might often appear to be less than that from $24 \mu \mathrm{m}$ radiation. But it is very improbable that star formation in these massive $\left(\sim 10^{8} M_{\odot}\right)$ molecular disks would occur in globally coherent bursts.

Alternatively, the relative absence of $\mathrm{H} \beta$ emission could be understood if massive stars are not forming in these S0 galaxies; Calzetti (2007) notes that $\operatorname{SFR}(H \beta)$ drops by $\sim 5$ if the maximum stellar mass is decreased from 120 to $30 M_{\odot}$. However, the stellar initial mass function (IMF) may be radically altered in molecular disks in S0 galaxies. Typical surface densities for the disks observed by Young, Bureau \& Cappellari (2008), $100-200 M_{\odot} \mathrm{pc}^{-2}$, correspond to about $0.02-0.04 \mathrm{gm}$ $\mathrm{cm}^{-2}$ which is less than the threshold density $\sim 1 \mathrm{gm}$ $\mathrm{cm}^{-2}$ required by Krumholz and McKee (2008) for massive star formation.

The column depths perpendicular to these molecular disks correspond to hydrogen columns of about $10^{22}$ $\mathrm{cm}^{-2}$ for which the optical depth to dust absorption of $\mathrm{H} \beta$ is about ten. Therefore it is conceivable that $\operatorname{SFR}(\mathrm{H} \beta)$ is underestimated simply because ionizing massive stars form close to the central disk plane where line emission cannot escape. However, the $24 \mu \mathrm{m}$ residuals and therefore $\operatorname{SFR}(24 \mu \mathrm{m})$ correlate with the stellar $\mathrm{H} \beta$ index so at least some of the young stars with Balmer (absorption) lines must be visible. For this obscuration model to work it would be necessary for A and F stars to be dynamically excited away from the disk plane so their radiation can escape. Further insights about the missing optical line emission in S0 galaxies might be gained by observing emission lines further in the infrared, like for example $\operatorname{Br} \gamma$ (at $2.16 \mu \mathrm{m})$, which are less absorbed by dust.

\section{DISCUSSION}

The star formation rates in Table 2 found from $24 \mu \mathrm{m}$ emission using the $\operatorname{SFR}-\left(\lambda L_{\lambda}\right)_{24}$ relation derived by Calzetti et al. (2007) suggests that the SFR in SAURON elliptical galaxies is $\lesssim 0.02 M_{\odot} \mathrm{yr}^{-1}$ and may in fact be zero. However, many S0 galaxies in the SAURON sample have SFRs that exceed $0.02 M_{\odot} \mathrm{yr}^{-1}$ and extend up to almost $0.2 M_{\odot} \mathrm{yr}^{-1}$ for NGC 3032 .

Our results complement those of Young, Bendo \& Lucero (2008) who studied MIPS infrared emission from a sample of $\mathrm{E}$ and $\mathrm{S} 0$ galaxies known to be very rich in molecular gas $\left(\mathrm{M}\left(\mathrm{H}_{2}\right) \approx 1.5-64 \times 10^{8} M_{\odot}\right)$. They found, as we do, evidence of enhanced $24 \mu \mathrm{m}$ emission from many S0 galaxies and showed that some S0s are measurably extended at this wavelength having sizes comparable to CO molecular images. This suggests that dust emitting at $24 \mu \mathrm{m}$ is indeed associated with star formation in cold molecular disks. Three of the galaxies in the $\mathrm{H}_{2}$-rich sample of Young, Bendo \& Lucero (2008) are also in the SAURON sample - NGC 3032, 4459 and 4526 - having $\mathrm{M}\left(\mathrm{H}_{2}\right)=2.6,1.6$ and $4.0 \times 10^{8} M_{\odot}$ respectively (Table 1).

Combes, Young \& Bureau (2007) have detected CO emission from about $28 \%$ of $\mathrm{E}$ or $\mathrm{S} 0$ galaxies in the SAURON sample. Of the ten galaxies with $\mathrm{CO}$ detections shown in our Table 1, the majority (8) are S0 galaxies. They also find that $\mathrm{CO}$-rich $\mathrm{E}$ and $\mathrm{S} 0$ galaxies have stronger $\mathrm{H} \beta$ spectral indices. This is consistent with our finding that both $\mathrm{M}\left(\mathrm{H}_{2}\right)$ and the $\mathrm{H} \beta$ index correlate with non-stellar $24 \mu$ emission in $\mathrm{S} 0$ galaxies.

Finally, the referee of our paper suggested that we also consider an analysis of the mid and far-infrared data in our sample using the new two-dimensional kinematical classification of early-type galaxies proposed by Emsellem et al. (2007) which is based on SAURON data. This new classification scheme is based on a single parameter, $\lambda_{R} \equiv\langle R|V|\rangle /\left\langle R\left(V^{2}+\sigma^{2}\right)^{1 / 2}\right\rangle$, where $\mathrm{R}$ is the distance from the galactic center in the image plane, $V$ is 
the stellar radial velocity, $\sigma$ is the stellar velocity dispersion, and the two-dimensional averages are taken within the effective radius $R_{e}$. Galaxies with $\lambda_{R}>0.1$ are designated fast rotators and those having $\lambda_{R}<0.1$ are slow rotators. Our Spitzer-SAURON sample contains 9 slow and 22 fast rotators. We made alternative versions of Figures 1 - 12 using this new classification scheme and determined that all correlations and conclusions based on the figures presented here remain unchanged, although the E-like slow rotators are fewer in number and more sparsely distribued in the alternative figures. Nevertheless, some plots are marginally different. For example in the $\lambda_{R}$-versions of Figures 1-3, the slow rotators exhibit less scatter in the vertical direction, with fast rotators lying both above and below, but this may be a result of the smaller number of slow rotators (9) compared to E galaxies (19) in the ususal classification. Nevertheless, we do not include the alternative figures here because they contain no important new information. Furthermore, we think it may be premature to abandon the familiar morphological early-type classification scheme in terms of $\mathrm{E}$ and $\mathrm{S} 0$ galaxies since the new $\lambda_{R}$ classification parameter is currently unavailable for the vast majority of early-type galaxies.

\section{CONCLUSIONS}

Perhaps the most important conclusion from these Spitzer observations of infrared emission from early type SAURON galaxies is that there are significant differences between S0 and E galaxies in the relative content of dust and cold gas and star formation rates. Only a small subset of S0 galaxies appear to share with $\mathrm{E}$ galaxies the same correlations between the $24 \mu \mathrm{m}$ luminosity, the $\mathrm{H} \beta$ index, and molecular mass. Results from Sloan surveys of "early type" galaxies that combine S0 and E galaxies (e.g. Kaviraj et al. 2007) may therefore be misleading if applied only to E galaxies. It is likely that the source of cold gas in E and S0 galaxies is systematically quite different. Rotationally supported cold gas in S0 galaxies may be a relic of their previous incarnation as late type spirals.

The threshold for measurable star formation rates in the SAURON sample is about $0.02 M_{\odot} \mathrm{yr}^{-1}$. All S0 galaxies (except NGC 474, 1023, 7457 and 4382) exceed this value and all E galaxies (except NGC 4486 and possibly NGC 2768) have lower SFRs. We have shown that the $24 \mu \mathrm{m}$ luminosity is a good measure of the SFR and that it correlates well with other related parameters such as the $\mathrm{H} \beta$ index, the mean stellar age determined from
SAURON observations and the mass of molecular gas.

Positive residuals from the E galaxy correlation between $\lambda L_{\lambda}$ at $24 \mu \mathrm{m}$ and $L_{K s}$ represent the emission from interstellar dust alone and are a measure of the galactic star formation rate. It is remarkable that these residuals for S0 galaxies correlate so tightly with normalized far-IR luminosities $L_{60} / L_{K s}$ and $L_{170} / L_{K s}$. This suggests that the amount of dust, cold gas and old stars is rather finely tuned. The estimated mass loss rates from old stars in So galaxies are very nearly the same as the star formation rates derived from interstellar $24 \mu \mathrm{m}$ emission. This supports the notion that the mass of cold, dusty gas in some SAURON S0 galaxies is in an approximate steady state - gas is lost by star formation at nearly the same rate that new gas is ejected from the dominant old stellar population.

However, the $\mathrm{H} \beta$ emission line luminosity from $\mathrm{S} 0$ galaxies appears to be very low and unrelated to the star formation rate. (This is also true for the global $B-K s$ colors of S0 galaxies in our sample.) Star formation rates determined from the mid-IR luminosity $\left(\lambda L_{\lambda}\right)_{24}$ are often about an order of magnitude larger than those found from the $\mathrm{H} \beta$ emission line luminosity, using the standard SFR recipes. Perhaps star formation in cold gaseous disks in early type galaxies does not extend to massive OB stars because the disk column densities fall below the Krumholtz-McKee threshold of $\sim 1 \mathrm{gm} \mathrm{cm}^{-2}$. An alternative interpretation is that $\mathrm{OB}$ stars do indeed form but are deeply embedded in dust that absorbs $\mathrm{H} \beta$ line emission. If this is the explanation, radiation must still escape from slightly older A-F type stars since the star formation rate based on $24 \mu \mathrm{m}$ emission correlates with the Lick $\mathrm{H} \beta$ index in the spectra of young $\sim \mathrm{A}$ stars. These non-ionizing stars may be less concentrated toward the central plane of the dusty molecular disks, allowing their radiation to escape.

This work is based on observations made with the Spitzer Space Telescope, which is operated by the Jet Propulsion Laboratory, California Institute of Technology, under NASA contract 1407. Support for this work was provided by NASA through Spitzer Guest Observer grant RSA 1276023. Studies of the evolution of hot gas in elliptical galaxies at UC Santa Cruz are supported by a Spitzer Theory Grant and an NSF grant for which we are very grateful. Finally we thank the referee for thoughtful remarks.

\section{REFERENCES}

Bell, E. F. et al. 2003, ApJS, 149, 289

Calzetti, D., 2007 (arXiv:0707.0467)

Calzetti, D. et al. 2007, ApJ, 666, 870

Caon, N., Macchetto, D. \& Pastoriza, M. 2000, ApJS 127, 39

Combes, F., Young, L. M. \& Bureau, M. 2007, MNRAS, 377, 1795

de Zeeuw, P. T. et al., 2002, MNRAS, 329, 513

Emsellem, E., et al. 2007, MNRAS, 379, 401

Kennicutt, R. C. 1998,ARA\&A, 36, 189

Krumholtz, M. R. \& McKee, C. F. 2008, Nature, 451, 1082

Kuntschner, H., et al. 2006, MNRAS, 369, 497

Mathews, W. G. 1989, AJ, 97, 42

McDermid, R.M. et al. 2006, MNRAS, 373, 906

Morganti, R., et al. 2006, MNRAS, 371, 157
Morganti, D. Roby, T., Khan, I., Booth, H. SPIE, 6274, 10

Rieke, G. H. 2004, ApJS, 154, 25

Sarzi, M. et al. 2006, MNRAS, 366, 1151

Temi, P., Brighenti, F. \& Mathews, W. G. 2008, ApJ, 672, 244

Temi, P., Brighenti, F. \& Mathews, W. G. 2007b, ApJ, 666, 222

Temi, P., Brighenti, F. \& Mathews, W. G. 2007a, ApJ, 660, 1215

Temi, P., Brighenti, F. \& Mathews, W. G. 2005, ApJ 635, L25

Tonry, J. L., et al. 2001, ApJ, 546, 681

Werner, M. W. et al. 2004, ApJS, 154, 1

Young, L. M., Bendo, G. L. \& Lucero, D. M. 2008, (arXiv:0803.4510

Young, L. M., Bureau, M. \& Cappellari, M. 2008, ApJ, 676. 317 
TABLE 1

\begin{tabular}{|c|c|c|c|c|c|c|c|c|c|c|c|}
\hline $\begin{array}{l}\text { Name } \\
(\text { NGC) }\end{array}$ & PI/Program ID & Type $^{\mathrm{a}}$ & $\mathrm{T}^{\mathrm{b}}$ & $\begin{array}{c}\mathrm{D}^{\mathrm{c}} \\
\mathrm{Mpc}\end{array}$ & $\begin{array}{c}\log L_{B} \\
\left(L_{B, \odot}\right)\end{array}$ & $\begin{array}{c}\log L_{K s}{ }^{\mathrm{d}} \\
\left(L_{K s, \odot}\right)\end{array}$ & $\begin{array}{l}\log M_{H I} \mathrm{e}^{\mathrm{e}} \\
\quad\left(M_{\odot}\right)\end{array}$ & $\begin{array}{c}\log M_{H_{2}}{ }^{f} \\
\quad\left(M_{\odot}\right)\end{array}$ & $\begin{array}{c}H \beta^{\mathrm{g}} \\
(\AA)\end{array}$ & $\begin{array}{l}\text { Age h } \\
\text { (Gyr) }\end{array}$ & $\begin{array}{c}\log F_{H \beta}{ }^{\mathrm{i}} \\
\left(\operatorname{erg~s}^{-1} \mathrm{~cm}^{2}\right)\end{array}$ \\
\hline 0821 & Fabbiano/20371 & E6? & -4.8 & 24.09 & 10.28 & 10.95 & $\cdots$ & & 1.53 & $\cdots$ & $\ldots$ \\
\hline 2768 & Temi/20171 & E6: & -4.3 & 22.38 & 10.63 & 11.25 & 8.23 & 7.65 & 1.70 & 2.5 & -13.55 \\
\hline 3377 & Fazio/69 & E5-6 & -4.8 & 11.22 & 9.82 & 10.47 & $\ldots$ & $<6.88$ & 1.85 & $\cdots$ & -13.63 \\
\hline 3379 & Fazio/69 & E1 & -4.8 & 10.57 & 10.11 & 10.89 & $\ldots$ & $<6.69$ & 1.43 & 11.5 & -14.41 \\
\hline 3608 & Fazio/30318 & $\mathrm{E} 2$ & -4.8 & 22.91 & 10.24 & 10.83 & $\ldots$ & $<7.51$ & 1.49 & 8.9 & -14.77 \\
\hline 4278 & Fazio/69 & E1-2 & -4.8 & 16.07 & 10.23 & 10.88 & 8.84 & 7.50 & 1.23 & $\ldots$ & -12.86 \\
\hline 4473 & Cote'/3649 & E5 & -4.7 & 15.71 & 10.13 & 10.88 & $\ldots$ & $<7.01$ & 1.49 & 13.2 & $\ldots$ \\
\hline 4486 & Cote'/3649 & $E 1^{+} p e c$ & -4.3 & 16.07 & 10.86 & 11.44 & $\ldots$ & $<7.03$ & 1.14 & 15.1 & -13.26 \\
\hline 4552 & Kennicutt/159 & E0-1 & -4.6 & 15.34 & 10.26 & 11.03 & $\ldots$ & $<7.18$ & 1.39 & 8.9 & -14.27 \\
\hline 4564 & Fabbiano/20371 & $\mathrm{E}$ & -4.8 & 15.00 & 9.81 & 10.53 & $\ldots$ & $\ldots$ & 1.52 & 3.6 & $\ldots$ \\
\hline 4621 & Cote'/3649 & E5 & -4.8 & 18.28 & 10.44 & 11.17 & $\ldots$ & $\ldots$ & 1.40 & $\ldots$ & $\ldots$ \\
\hline 4660 & Cote'/3649 & $\mathrm{E}$ & -4.7 & 12.82 & 9.55 & 10.28 & $\ldots$ & $\ldots$ & 1.43 & $\ldots$ & $\ldots$ \\
\hline 5813 & Fazio/69 & E1-2 & -4.8 & 32.21 & 10.73 & 11.21 & $\ldots$ & $<7.64$ & 1.51 & 15.1 & -14.01 \\
\hline 0474 & Zezas/20140 & $S 0^{0}(s)$ & -2.0 & 33.46 & 10.36 & 10.77 & $\ldots$ & $<7.63$ & 1.68 & $\ldots$ & -14.09 \\
\hline 1023 & Fazio/69 & $S B 0^{-}(r s)$ & -2.7 & 11.42 & 10.59 & 10.96 & 9.32 & $<6.69$ & 1.49 & 4.7 & -14.1 \\
\hline 2685 & Rieke/40936 & (R) $S B 0^{+} p e c$ & -1.1 & 15.63 & 9.83 & 10.41 & 9.26 & 7.50 & 2.01 & $\ldots$ & -13.48 \\
\hline 3032 & Young/20780 & $S A B 0^{0}(r)$ & -1.8 & 21.98 & 9.69 & 10.17 & $\ldots$ & 8.42 & 4.57 & 0.9 & -13.79 \\
\hline 3156 & Surace/3403 & S0: & -2.4 & 22.39 & 9.81 & 10.23 & $\ldots$ & 7.62 & 3.92 & $\cdots$ & -13.71 \\
\hline 3384 & Surace/3403 & $S B 0^{-}(s)$ & -2.7 & 11.59 & 9.98 & 10.78 & $\ldots$ & $<7.07$ & 1.94 & 3.2 & -14.67 \\
\hline 3489 & Fazio/69 & $S A B 0^{+}(r s)$ & -1.3 & 12.08 & 9.96 & 10.54 & $\ldots$ & 7.19 & 2.82 & 1.7 & -12.95 \\
\hline 4150 & Fazio/69 & $S 0^{0}(r)$ & -2.1 & 13.74 & 10.30 & 10.02 & 6.40 & 7.82 & 3.47 & 1.5 & -13.9 \\
\hline 4382 & Cote'/3649 & $\mathrm{SO}^{+}(\mathrm{s}) \mathrm{pec}$ & -1.3 & 18.45 & 10.77 & 11.70 & $\ldots$ & $<7.34$ & 2.16 & 1.7 & $\ldots$ \\
\hline 4459 & Cote'/3649 & $S 0^{+}(r)$ & -1.4 & 16.14 & 10.11 & 10.81 & $\ldots$ & 8.22 & 2.16 & 1.9 & -14.07 \\
\hline 4477 & Rieke/40936 & SB0(s): & -1.9 & 17.06 & 10.19 & 10.87 & $\ldots$ & 7.55 & 1.65 & $\ldots$ & -13.54 \\
\hline 4526 & Fazio/69 & $S A B 0^{\circ}(s)$ : & -1.9 & 16.90 & 10.52 & 11.21 & $\ldots$ & 8.60 & 1.86 & 2.8 & -13.74 \\
\hline 4570 & Cote'/3649 & S0 sp & -2.0 & 25.90 & 10.37 & 10.73 & $\ldots$ & $<7.75$ & 1.45 & $\ldots$ & -16.02 \\
\hline 7457 & Fazio/30318 & $S 0^{-}(r s) ?$ & -2.6 & 13.24 & 9.97 & 10.32 & $<6.18$ & 6.66 & 2.28 & $\ldots$ & -14.49 \\
\hline
\end{tabular}

${ }^{a}$ Hubble type is taken from de Zeeuw et al. (2002). ${ }^{b}$ The morphological type T is taken from the HyperLeda database. ${ }^{c}$ Distances are taken from Tonry et al. (2001). ${ }^{d}$ Luminosities in the $K s$ band have been derived using the flux density $F_{K s}$ published by the 2 MASS survey. ${ }^{e}$ The neutral hydrogen masses $M_{H I}$ are taken from Morganti et al. (2006). ${ }^{f}$ Molecular gas masses $M_{H_{2}}$ are taken from Combes et al (2007). ${ }^{g} H \beta$ indexes (estimated within $R_{e} / 8$ ) from Kuntschner et al. (2006). ${ }^{h}$ Ages are taken from McDermid et al. (2006). ${ }^{i} H \beta$ emission fluxes are taken from Sarzi et al. (2006) 
Temi, Brighenti \& Mathews

TABLE 2

\begin{tabular}{|c|c|c|c|c|c|c|c|}
\hline \multirow{2}{*}{$\begin{array}{l}\text { Name } \\
(\mathrm{NGC})\end{array}$} & \multicolumn{3}{|c|}{ Flux Density } & \multicolumn{3}{|c|}{$\log L_{\lambda} \Delta \lambda$} & \multirow{2}{*}{$\begin{array}{c}\mathrm{SFR}^{\mathrm{a}} \\
(24 \mu \mathrm{m}) \\
\left(M_{\odot} y r^{-1}\right)\end{array}$} \\
\hline & $\begin{array}{l}24 \mu \mathrm{m} \\
(\mathrm{mJy})\end{array}$ & $\begin{array}{l}70 \mu \mathrm{m} \\
(\mathrm{mJy})\end{array}$ & $\begin{array}{r}160 \mu \mathrm{m} \\
(\mathrm{mJy})\end{array}$ & $\begin{array}{r}24 \mu \mathrm{m} \\
\left(\operatorname{erg~s}^{-1}\right)\end{array}$ & $\begin{array}{r}70 \mu \mathrm{m} \\
\left(\operatorname{erg~s}^{-1}\right)\end{array}$ & $\begin{array}{r}160 \mu \mathrm{m} \\
\left(\operatorname{erg~s}^{-1}\right)\end{array}$ & \\
\hline 0821 & $15.1 \pm 4.8$ & $<15.6$ & $<24.8$ & 40.46 & $<40.10$ & $<39.84$ & $\ldots$ \\
\hline 2768 & $46.6 \pm 8.2$ & $694 \pm 26$ & $377 \pm 29$ & 40.89 & 41.68 & 40.95 & $2.03 \times 10^{-2}$ \\
\hline 2974 & $62.5 \pm 12.4$ & $682 \pm 18$ & $1979 \pm 54$ & 40.98 & 41.64 & 41.64 & $\ldots$ \\
\hline 3377 & $17.3 \pm 5.4$ & $80.6 \pm 5.7$ & $70.7 \pm 9.2$ & 39.86 & 40.15 & 39.63 & $\ldots$ \\
\hline 3379 & $65.5 \pm 8.8$ & $60.5 \pm 8.0$ & $59.1 \pm 8.2$ & 40.38 & 39.97 & 39.50 & $\ldots$ \\
\hline 3608 & $18.4 \pm 4.2$ & $66.2 \pm 8.2$ & $94 \pm 21$ & 40.50 & 40.68 & 40.37 & $1.18 \times 10^{-2}$ \\
\hline 4278 & $43.9 \pm 12.7$ & $790 \pm 18$ & $1356 \pm 28$ & 40.57 & 41.45 & 41.22 & $1.49 \times 10^{-2}$ \\
\hline 4374 & $65.3 \pm 8.6$ & $588 \pm 21$ & $487 \pm 21$ & 40.86 & 41.44 & 40.89 & $\ldots$ \\
\hline 4458 & $3.2 \pm 1.1$ & $<16.5$ & $<21.8$ & 39.50 & $<39.83$ & $<39.49$ & $\ldots$ \\
\hline 4473 & $25.8 \pm 6.7$ & $<8.0$ & $<15.1$ & 40.32 & 39.44 & 39.25 & $\ldots$ \\
\hline 4486 & $151 \pm 9$ & $460 \pm 14$ & $815 \pm 31$ & 41.11 & 41.22 & 41.00 & $4.03 \times 10^{-2}$ \\
\hline 4552 & $57.3 \pm 7.8$ & $92.1 \pm 10.2$ & $171 \pm 16$ & 40.65 & 40.48 & 40.28 & $1.08 \times 10^{-2}$ \\
\hline 4564 & $23.5 \pm 3.7$ & & & 40.24 & & $\ldots$ & $\ldots$ \\
\hline 4621 & $34.2 \pm 6.3$ & $31.7 \pm 5.7$ & $43.4 \pm 6.6$ & 40.58 & 40.17 & 39.84 & $\ldots$ \\
\hline 4660 & $15.2 \pm 4.3$ & $36.4 \pm 6.2$ & $53.7 \pm 8.2$ & 39.92 & 39.92 & 39.62 & $2.93 \times 10^{-3}$ \\
\hline 5813 & $14.9 \pm 4.9$ & $58.4 \pm 7.6$ & $34.8 \pm 5.9$ & 40.71 & 40.93 & 40.24 & $\ldots$ \\
\hline 5831 & $14.5 \pm 4.6$ & $<15.8$ & $<21.6$ & 40.55 & $<40.21$ & $<39.88$ & $1.46 \times 10^{-2}$ \\
\hline 5845 & $7.6 \pm 3.2$ & $103 \pm 12$ & $154 \pm 13$ & 40.23 & 40.98 & 40.69 & $\ldots$ \\
\hline 5846 & $38.5 \pm 6.2$ & $102 \pm 10$ & $117 \pm 12$ & 40.90 & 40.94 & 40.54 & $3.65 \times 10^{-3}$ \\
\hline 5982 & $13.5 \pm 5.1$ & $37.2 \pm 7.2$ & $59.9 \pm 11.7$ & 40.86 & 40.92 & 40.66 & $7.17 \times 10^{-3}$ \\
\hline 0474 & $4.9 \pm 2.2$ & $36.2 \pm 7.5$ & $97.6 \pm 22.6$ & 40.26 & 40.75 & 40.72 & $\cdots$ \\
\hline 1023 & $58.9 \pm 5.6$ & $<15.6$ & $<24.8$ & 40.40 & $<39.45$ & $<39.19$ & $\ldots$ \\
\hline 2685 & & & & & & $\ldots$ & $\ldots$ \\
\hline 3032 & $149 \pm 8$ & $2640 \pm 34$ & $2768 \pm 73$ & 41.38 & 42.25 & 41.80 & $1.97 \times 10^{-1}$ \\
\hline 3156 & $16.3 \pm 6.2$ & $242 \pm 8$ & $185 \pm 11$ & 40.43 & 41.23 & 40.64 & $2.41 \times 10^{-2}$ \\
\hline 3384 & $60.2 \pm 7.8$ & $42.5 \pm 17.2$ & $\ldots$ & 40.43 & 39.90 & $\ldots$ & $\ldots$ \\
\hline 3489 & $89.8 \pm 6.7$ & $1673 \pm 18$ & $2559 \pm 38$ & 40.64 & 41.53 & 41.25 & $3.38 \times 10^{-2}$ \\
\hline 4150 & $66.9 \pm 9.5$ & $1432 \pm 28$ & $1672 \pm 35$ & 40.62 & 41.57 & 41.18 & $4.02 \times 10^{-2}$ \\
\hline 4382 & $51.1 \pm 6.7$ & $22.5 \pm 6.5$ & $18.7 \pm 9.1$ & 40.76 & 40.03 & 39.48 & \\
\hline 4459 & $105 \pm 8$ & $2286 \pm 19$ & $3147 \pm 43$ & 40.96 & 41.92 & 41.59 & $6.69 \times 10^{-2}$ \\
\hline 4477 & $12.6 \pm 3.2$ & $<18$ & $<22$ & 40.08 & $<39.86$ & $<39.48$ & \\
\hline 4526 & $262 \pm 12$ & $7750 \pm 32$ & $12460 \pm 67$ & 41.39 & 42.49 & 42.23 & $1.68 \times 10^{-1}$ \\
\hline 4570 & $18.3 \pm 6.0$ & $<18.3$ & $<27.6$ & 40.61 & 40.22 & 39.94 & $2.50 \times 10^{-2}$ \\
\hline 7457 & $8.2 \pm 2.5$ & $<15.9$ & $<23.5$ & 39.68 & $<39.59$ & $<39.29$ & $\ldots$ \\
\hline
\end{tabular}




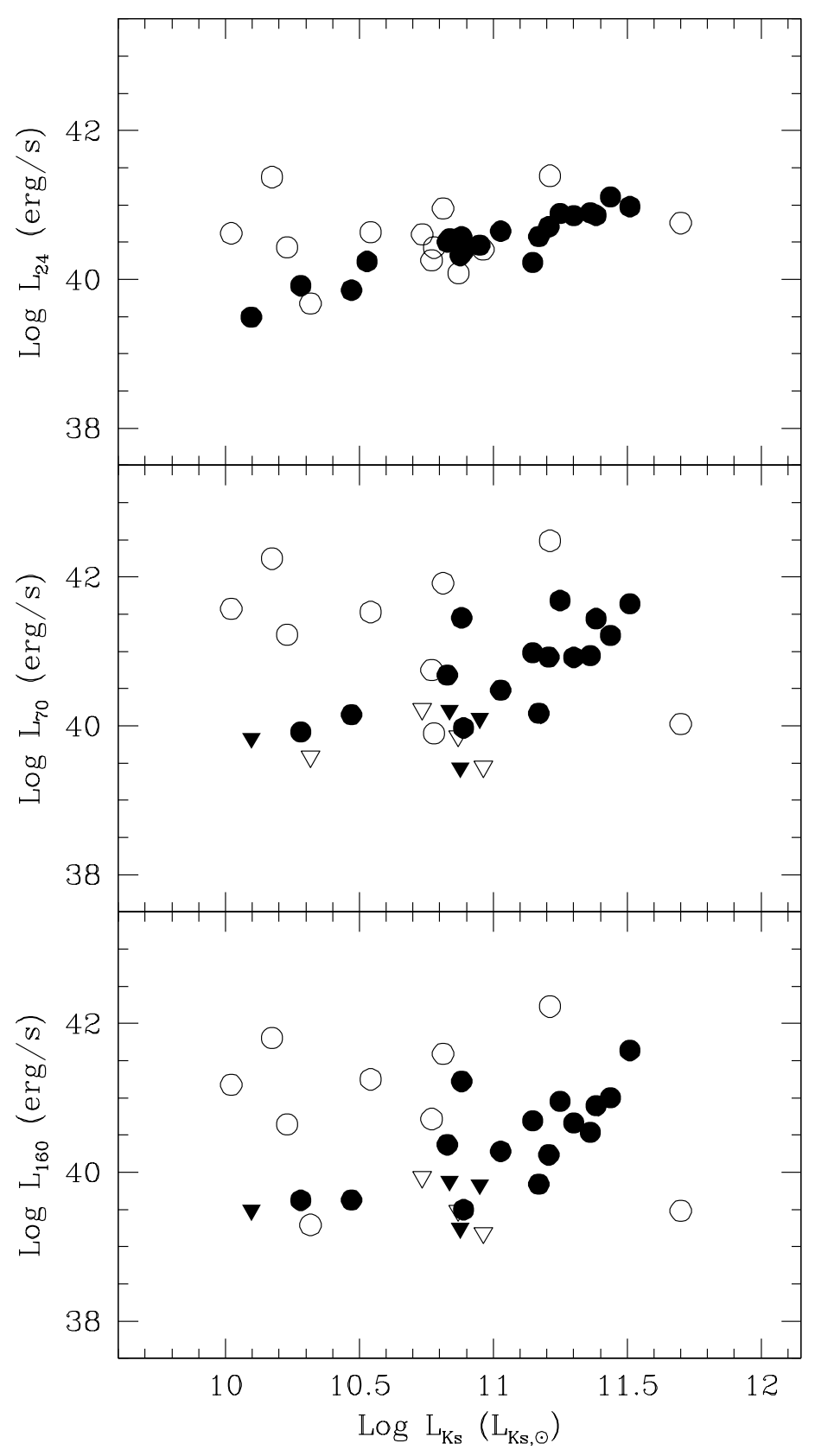

FIG. 1.- SAURON galaxy luminosities in 24, 60 and $170 \mu \mathrm{m}$ Spitzer bandpasses plotted against 2MASS Ks band luminosities. Detected E galaxies are filled circles and S0 galaxies are open circles. Triangles represent upper limits. 


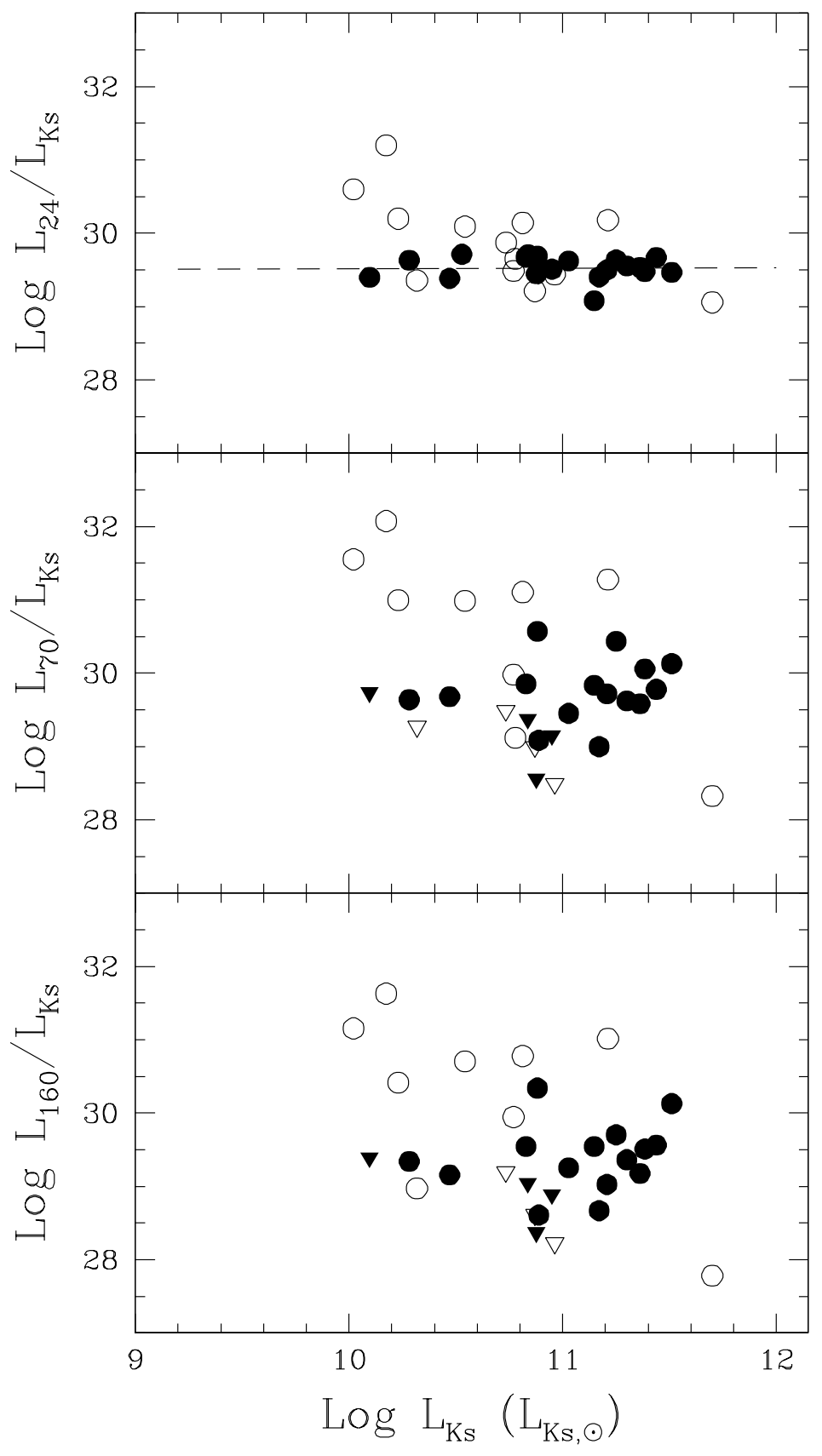

Fig. 2.- Plot similar to Figure 1 but with $L_{F I R} / L_{K s}$ plotted against $L_{K s}$. The dashed line in the upper panel is a least squares fit for just the E galaxies (filled circles). The $\mathrm{Ks}-24 \mu \mathrm{m}$ color is nearly constant for old stellar populations in E galaxies regardless of $L_{K s}$. In general S0 galaxies (open circles) have a much wider range of $L_{F I R}$ than $\mathrm{E}$ galaxies. 


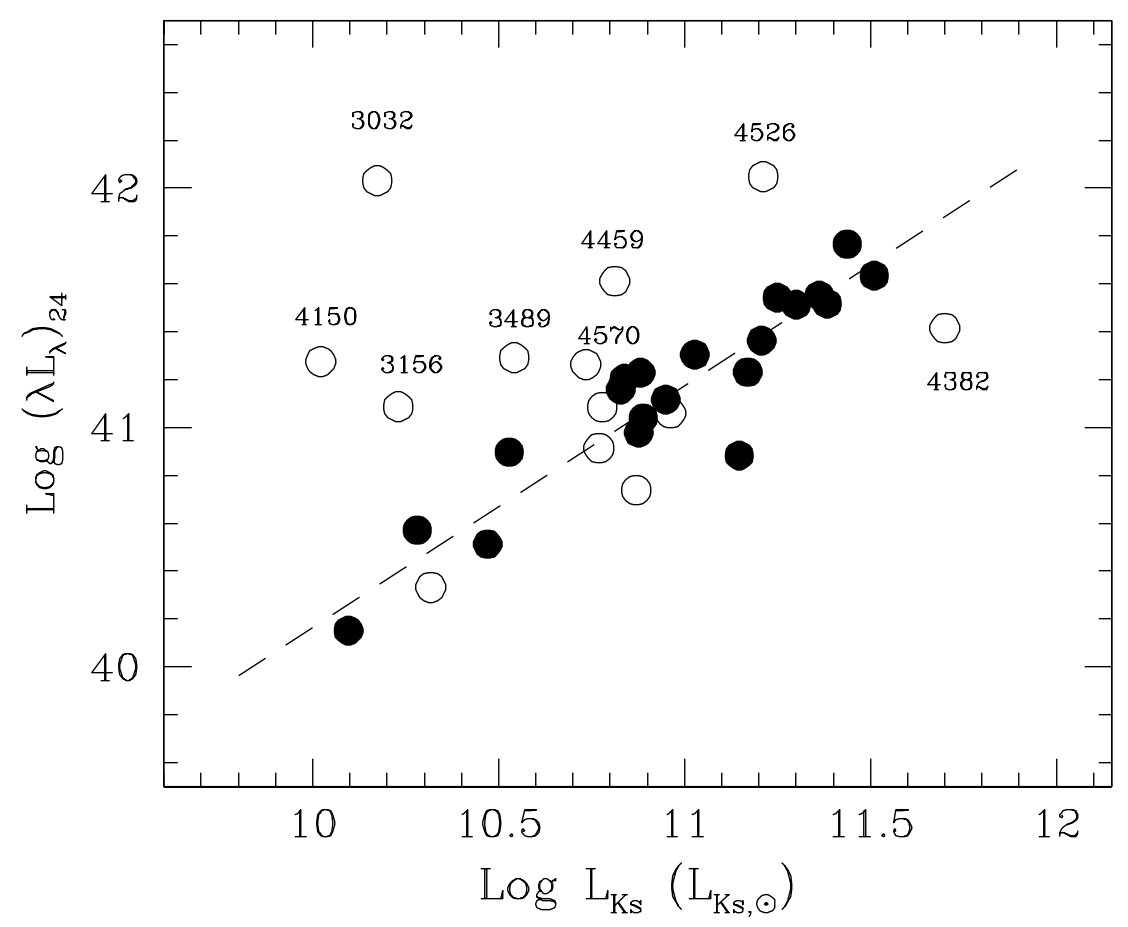

FIG. 3.- Plot of $\left(\lambda L_{\lambda}\right)_{24}$ against Ks-band luminosity $L_{K s}$, showing a tight correlation among E galaxies and uncorrelated $24 \mu$ excesses for many S0 galaxies. 


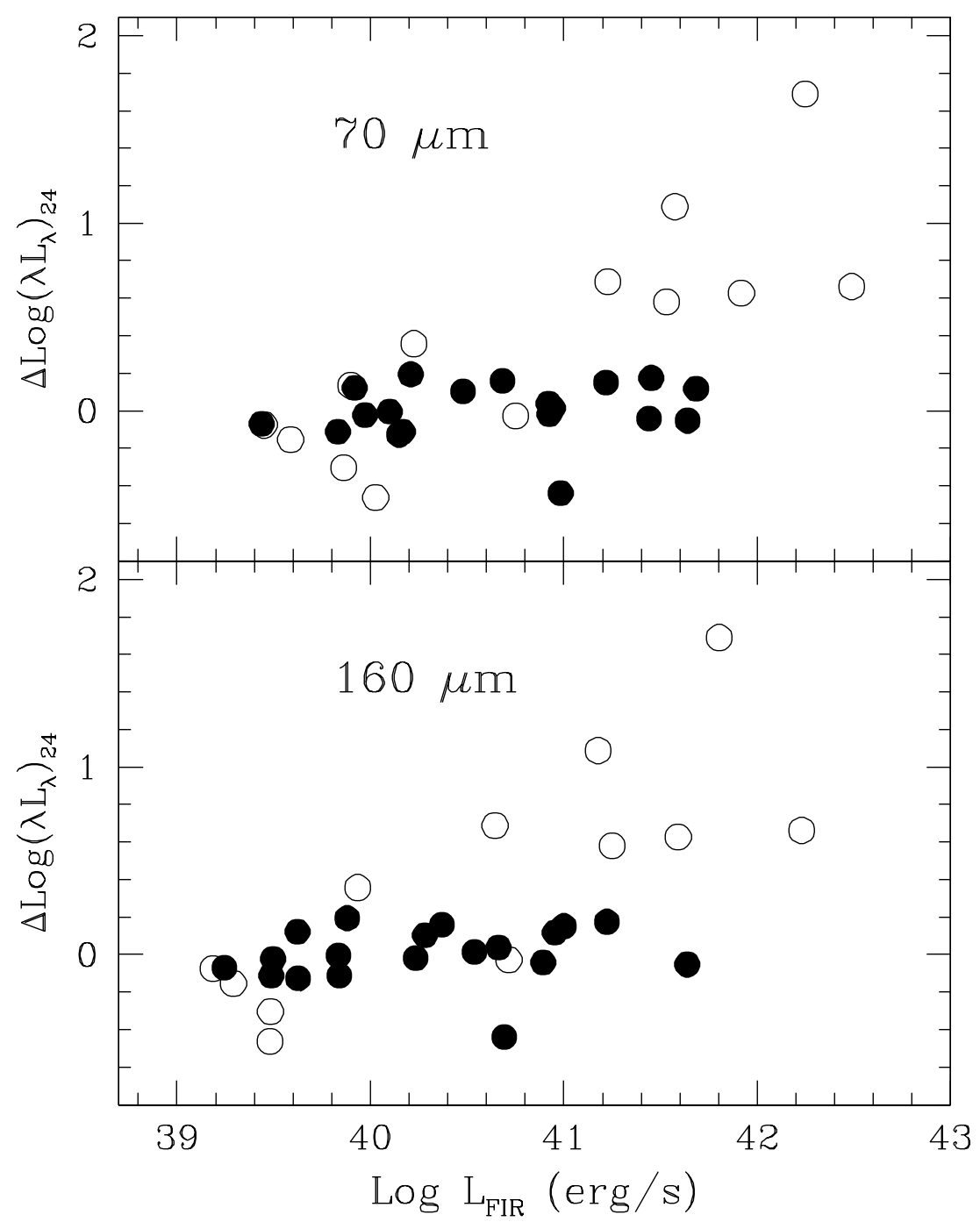

FIG. 4.- Residuals $\Delta \log \left(\lambda L_{\lambda}\right)_{24}$ in Figure 3 plotted against passband luminosities at 70 and $160 \mu \mathrm{m}$. In this and subsequent figures $L_{F I R}$ represents either $L_{70}$ or $L_{160}$. 


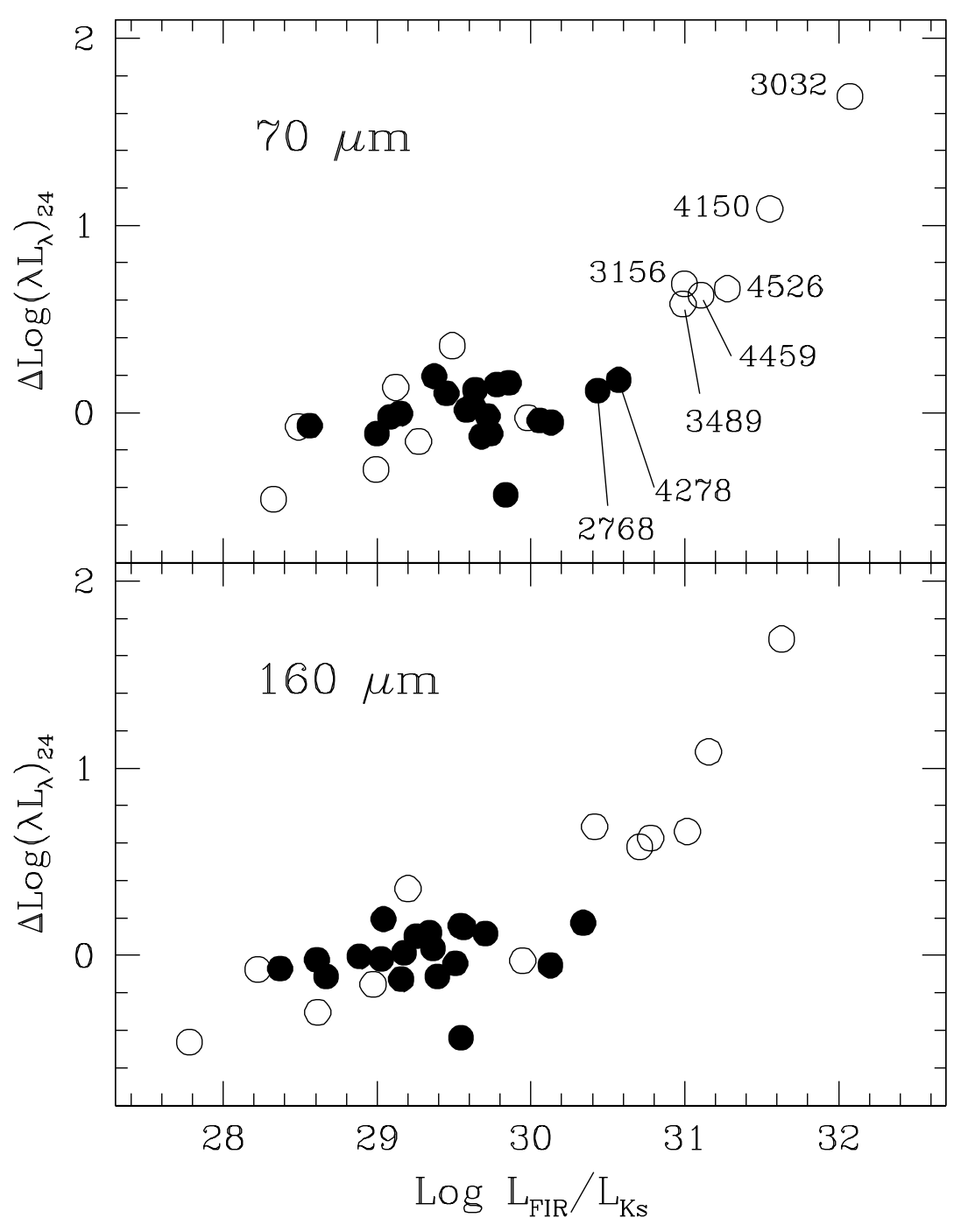

FIG. 5.- Residuals $\Delta \log \left(\lambda L_{\lambda}\right)_{24}$ in Figure 3 plotted against passband luminosities at 70 and $160 \mu$ m normalized with $L_{K s}$. Note that the correlation is much tighter than in Figure 4. 


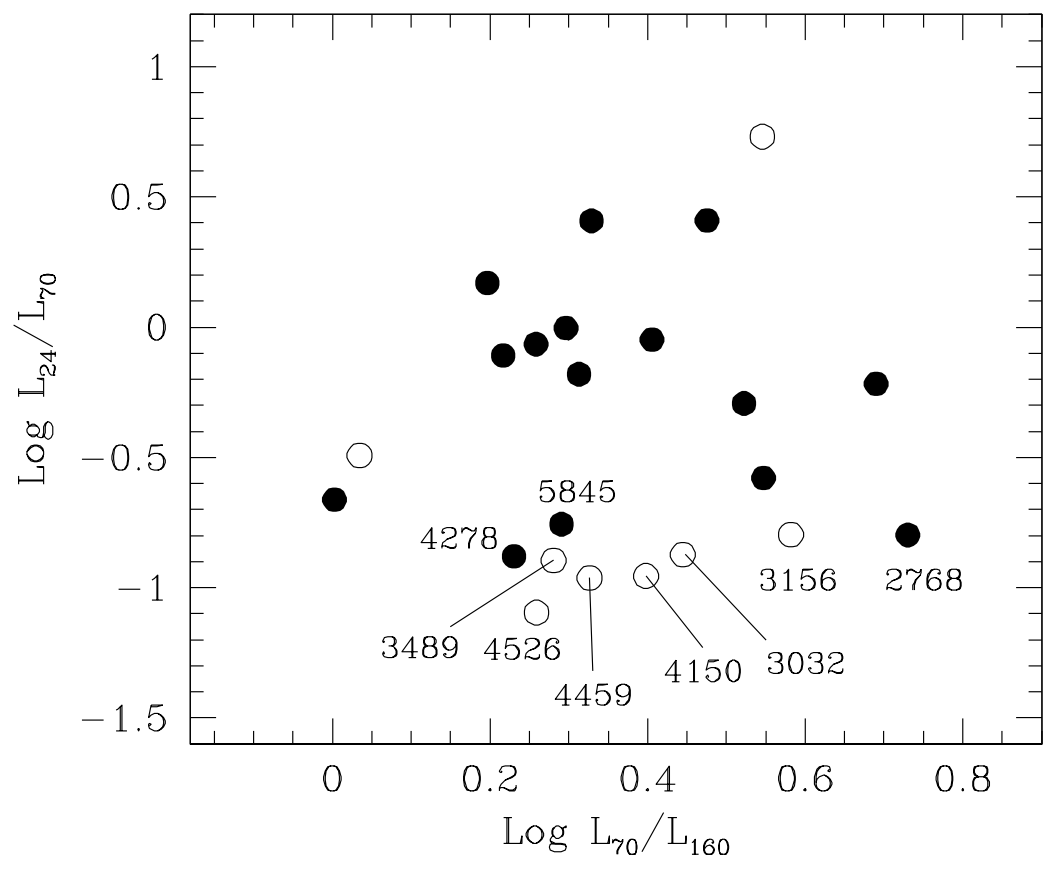

FIG. 6.- Infrared color-color plot for 24, 70 and $160 \mu \mathrm{m}$ emission from Spitzer-SAURON galaxies. Labeled galaxies with known molecular disks lie systematically near the bottom of the plot with small $\log \left(L_{24} / L_{70}\right)$. 


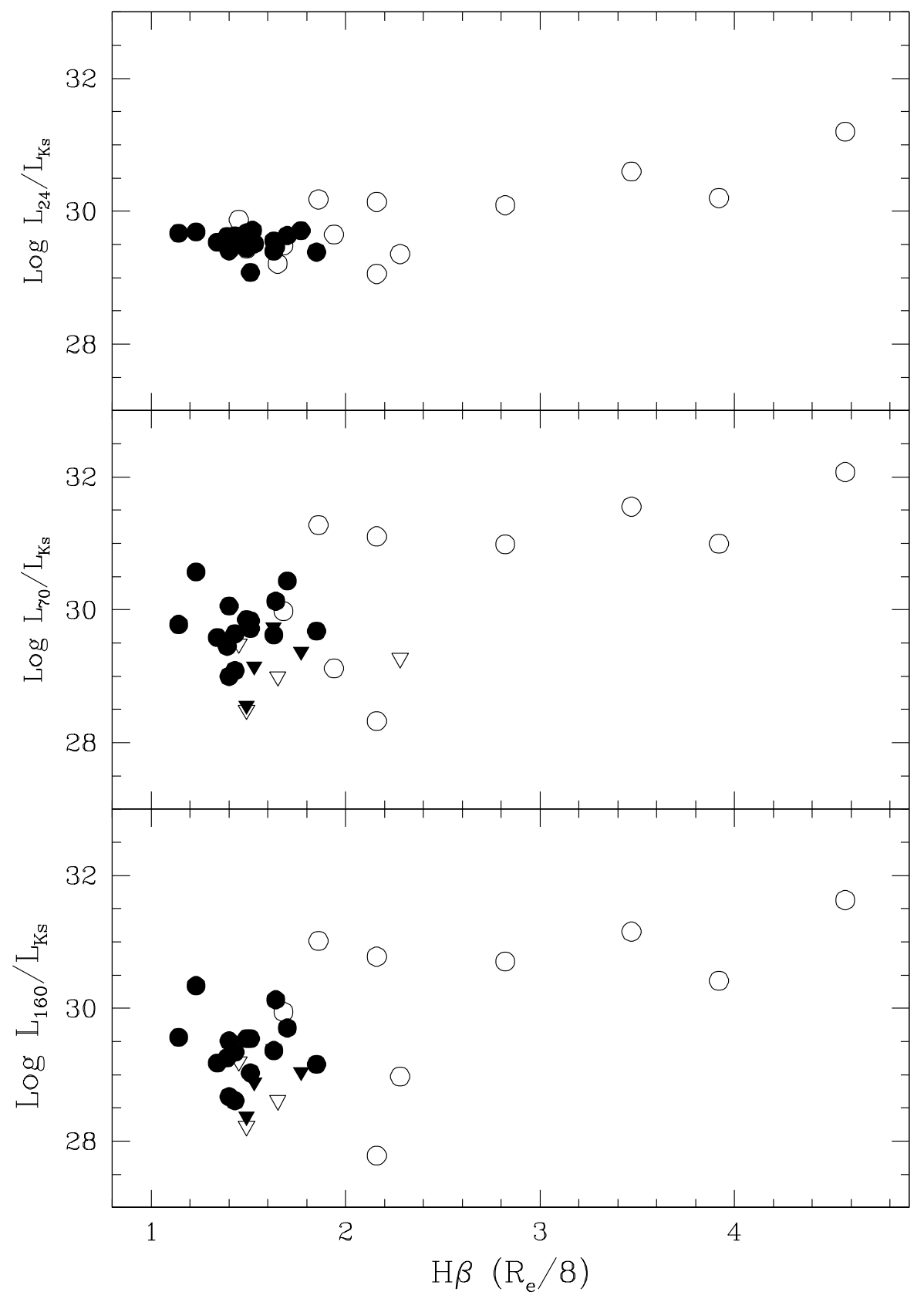

FIG. 7.- Variation of $L_{F I R} / L_{K s}$ with stellar $\mathrm{H} \beta$ index (within $R_{e} / 8$ ). The Pearson correlation coefficients for the S0 galaxies at 24 , 70 , and $160 \mu \mathrm{m}$ are $r_{S 0}=0.77,0.61$ and 0.46 respectively (discounting upper limits). 

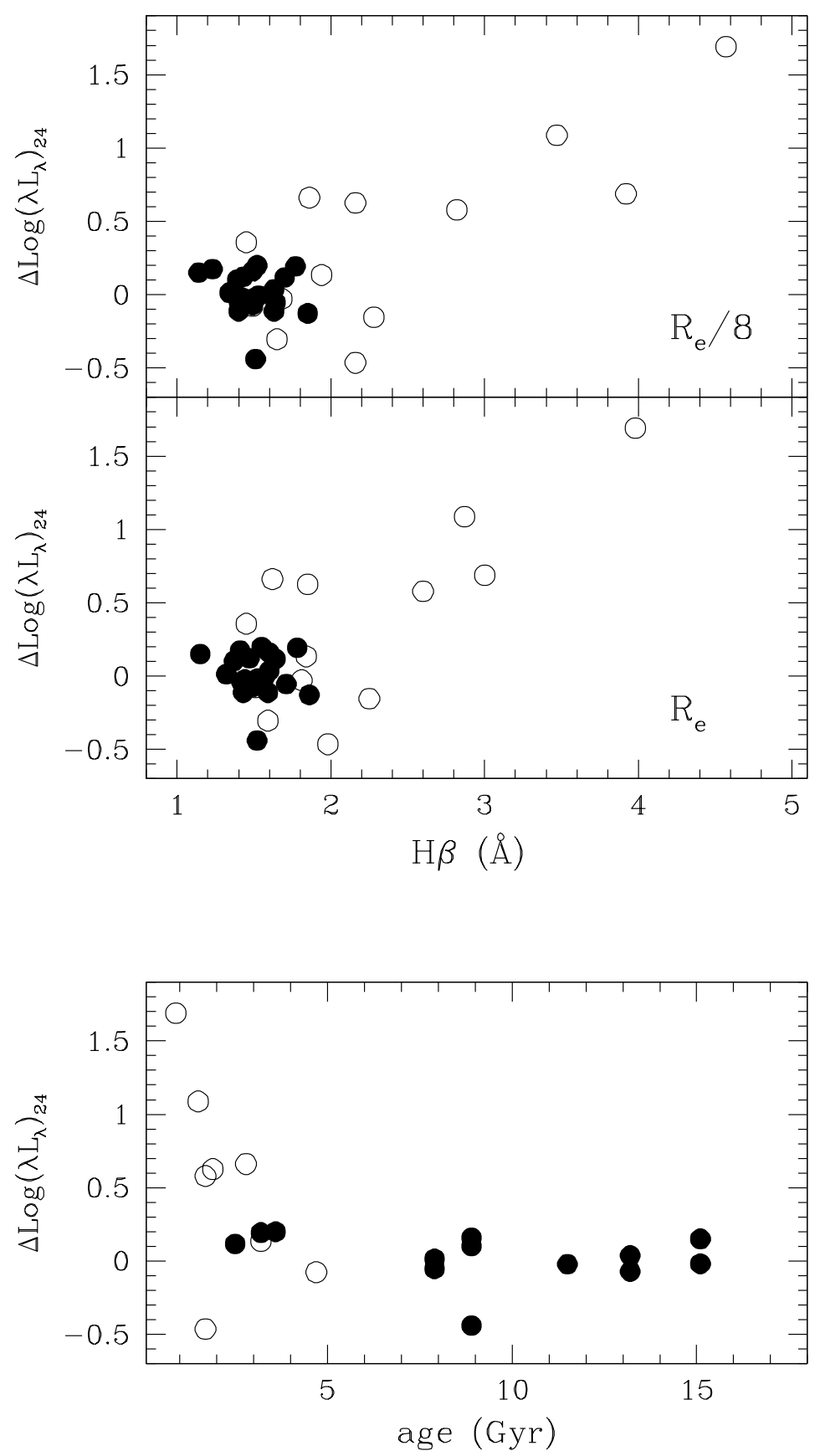

Fig. 8.- Upper panels: Residuals $\Delta \log \left(\lambda L_{\lambda}\right)_{24}$ in Figure 3 plotted against stellar $\mathrm{H} \beta$ index measured within $R_{e} / 8$ or $R_{e}$. Symbols as in Figure 1. The Pearson correlation coefficients for the S0 galaxies are $r_{S 0}=0.77$ for $R_{e} / 8$ and $r_{S 0}=0.75$ for $R_{e}$. Lower panel: Residuals $\Delta \log \left(\lambda L_{\lambda}\right)_{24}$ in Figure 3 plotted against luminosity-weighted stellar age from Table 1. 


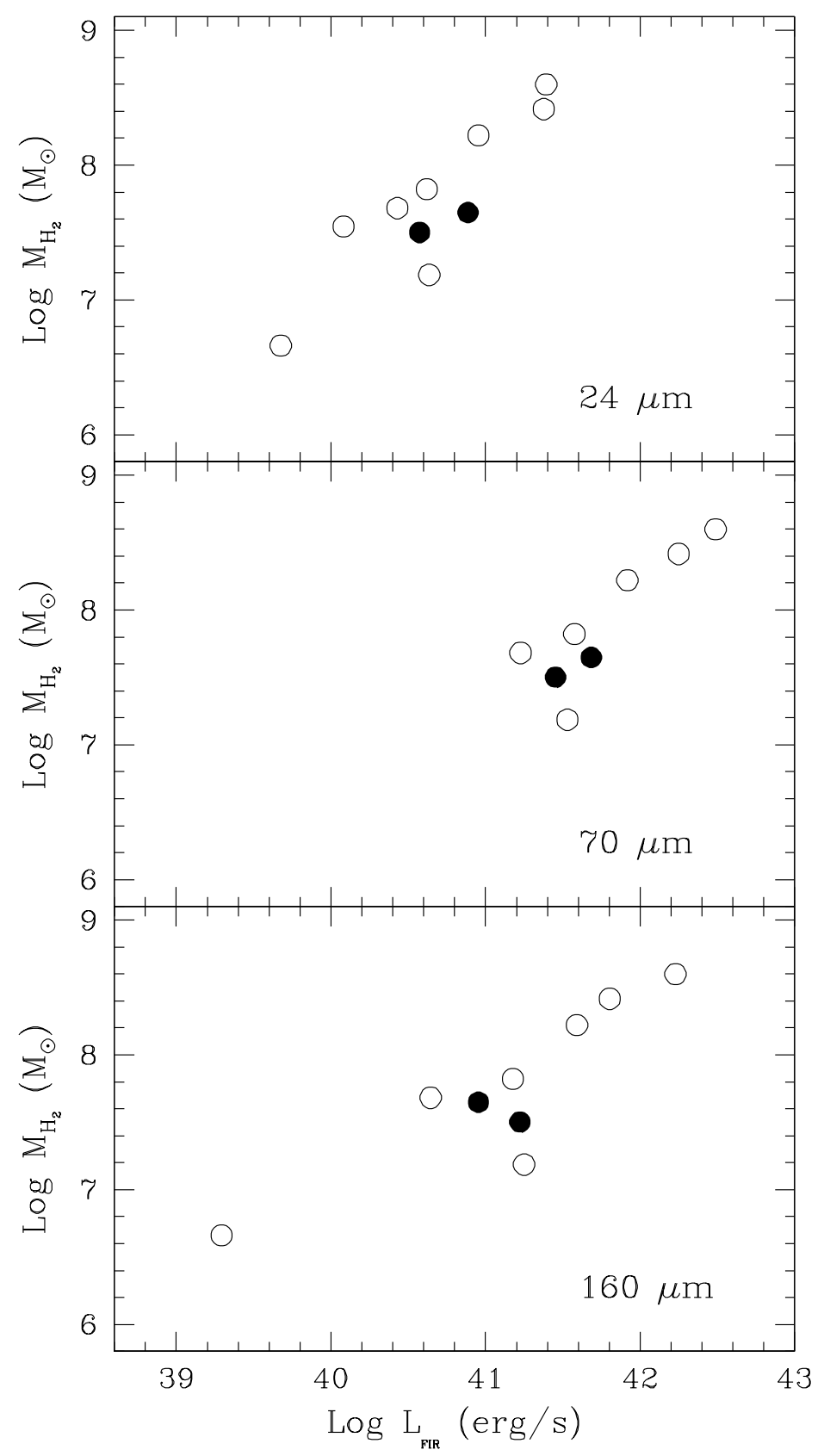

FIG. 9.- Variation of far-IR bandpass luminosities with mass of molecular gas. A positive correlation is seen at all three wavelengths, 24, 60 and $170 \mu \mathrm{m}$. Symbols as in Figure 1. 


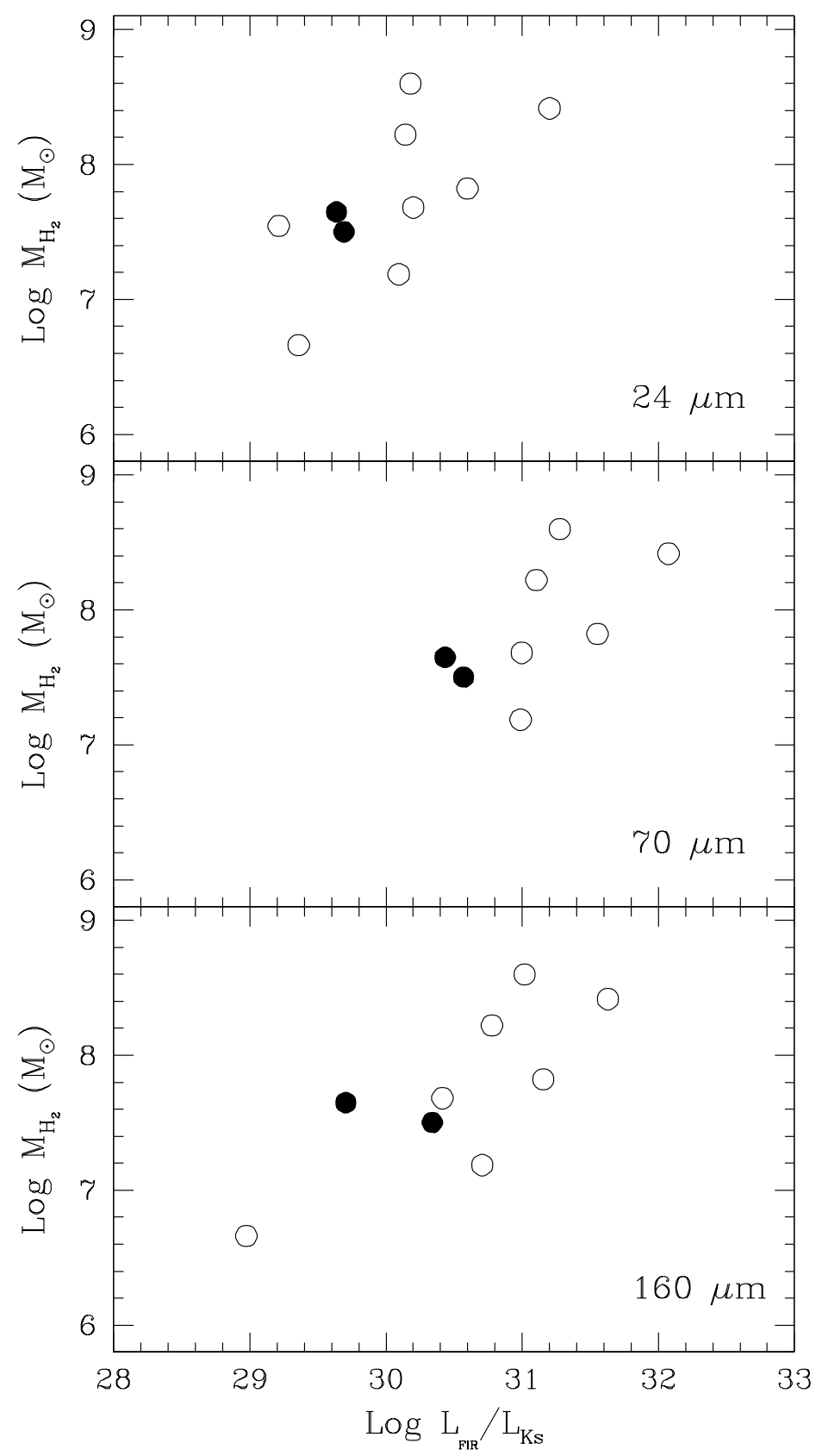

FIG. 10.- Plots similar to those in Figure 9 but with molecular mass plotted against far-IR luminosities normalized with $L_{K s}$. Symbols 


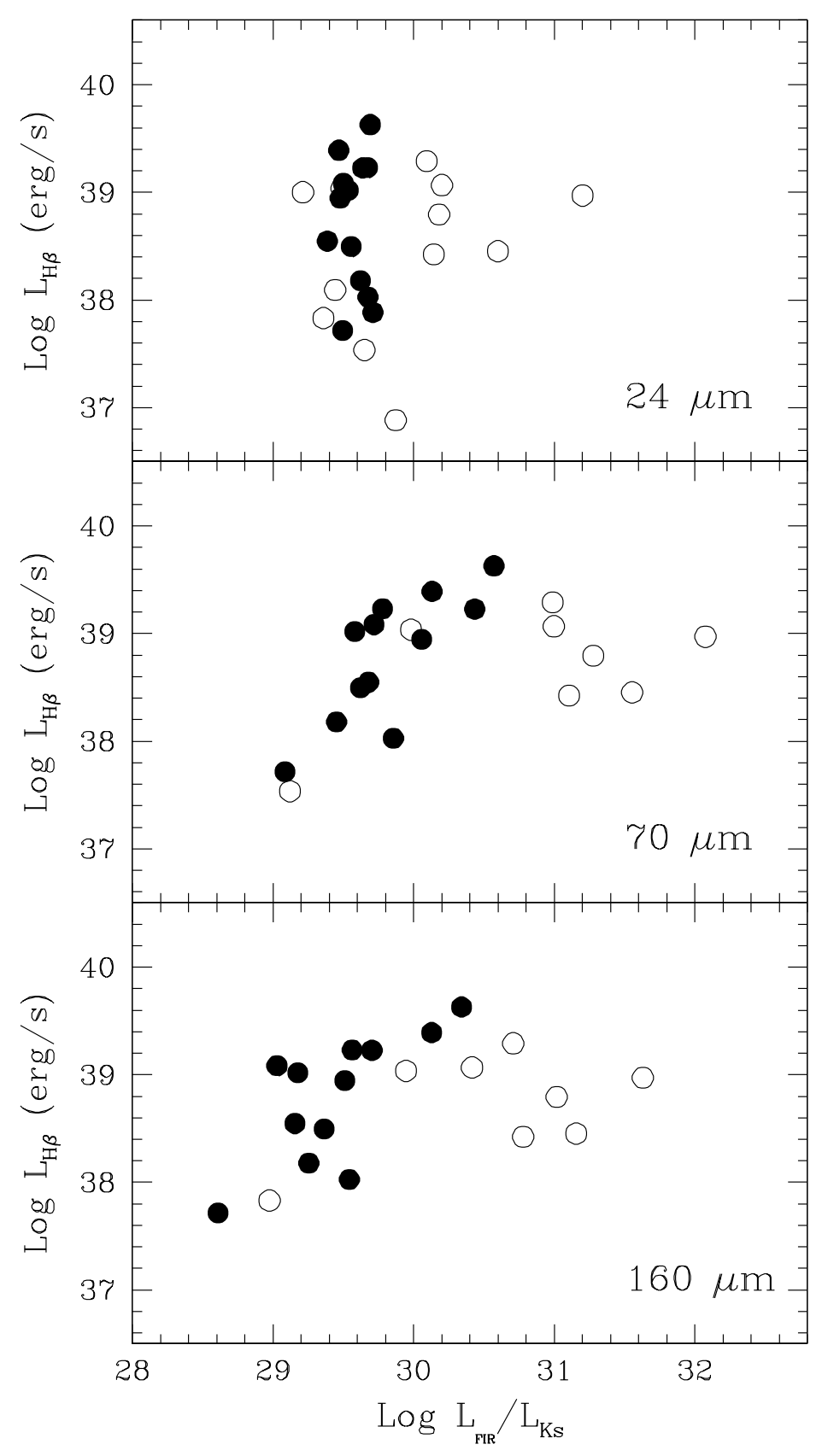

FIG. 11.- $\mathrm{H} \beta$ emission line luminosities plotted against far-IR band pass luminosities normalized with $L_{K s}$. E galaxies (filled circles) show correlations at 60 and $170 \mu \mathrm{m}$, but S0 data (open circles) show larger scatter at all three infrared wavelengths. The Pearson correlation coefficients at 24,70 and $160 \mu$ for $\mathrm{S} 0$ and E galaxies are respectively: $r_{S 0}=0.27,0.51 \& 0.32$ and $r_{E}=0.04,0.78$ and 0.72 . 

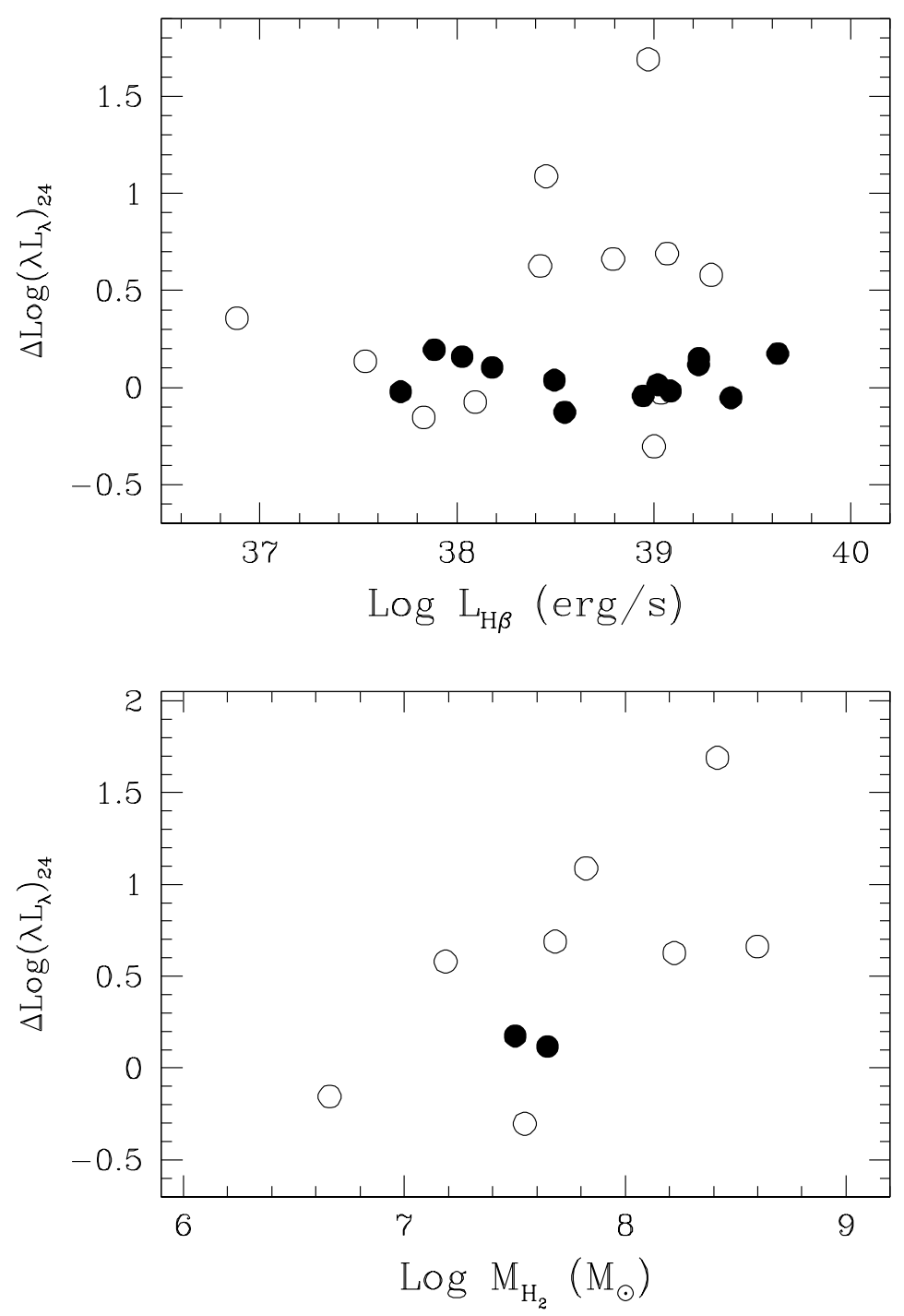

Fig. 12.- Upper panel: Residuals in Figure $3 \Delta \log \left(\lambda L_{\lambda}\right)_{24}$ plotted against $\mathrm{H} \beta$ luminosities. E galaxies are filled circles and S0 galaxies are open. Lower panel: Residuals in Figure 3 plotted against mass of molecular gas. E galaxies are filled circles and S0 galaxies are open. 


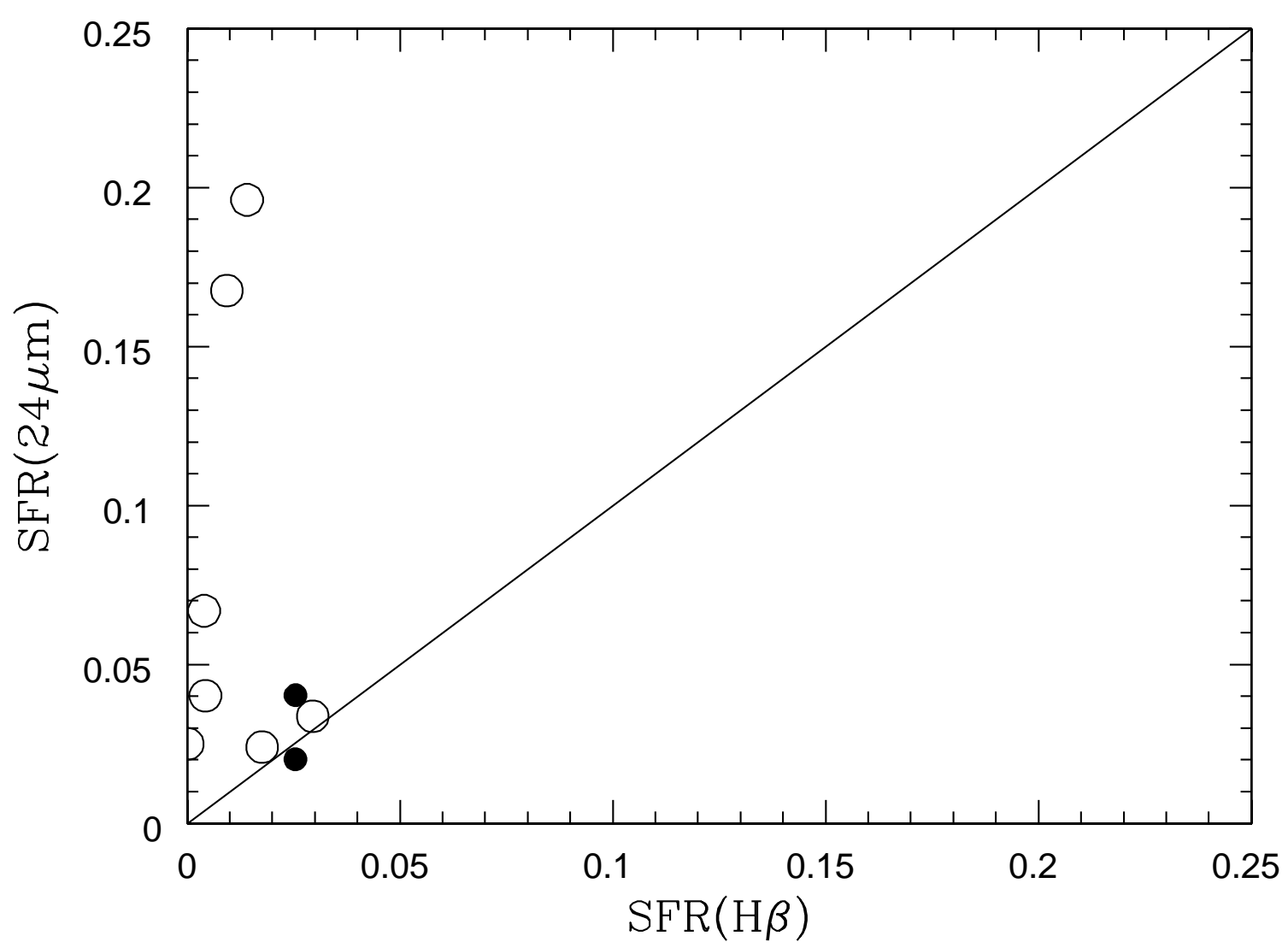

FIG. 13. - Estimated star formation rates from $24 \mu \mathrm{m}$ emission plotted against star formation rates estimated from $H \beta$ emission line luminosities. Most S0 galaxies (open circles) have $\operatorname{SFR}(\mathrm{H} \beta)<<\operatorname{SFR}(24 \mu \mathrm{m})$. Data is available for only 2 E galaxies (filled circles). 